This is the final peer-reviewed accepted manuscript of

VIOLA, IRENE; OPPO, DAVIDE; Franchi, F.; CAPOZZI, ROSSELLA; DINELLI, ENRICO; Liverani, B.; Taviani, M.:

Mineralogy, geochemistry and petrography of methane-derived authigenic carbonates from Enza River,

Northern Apennines (Italy). MARINE AND PETROLEUM GEOLOGY 66. ISSN 0264-8172

DOI: 10.1016/j.marpetgeo.2015.03.011

The final published version is available online at:

http://dx.doi.org/10.1016/j.marpetgeo.2015.03.011

Rights / License: The terms and conditions for the reuse of this version of the manuscript are specified in the publishing policy. For all terms of use and more information see the publisher's website. 


\title{
Mineralogy, geochemistry and petrography of methane-derived authigenic carbonates from Enza River, Northern Apennines (Italy)
}

\author{
I. Viola ${ }^{a,}{ }^{*}$, D. Oppo ${ }^{\text {a }}$, F. Franchi ${ }^{\text {b, c }}$, R. Capozzi ${ }^{\text {a }}$, E. Dinelli ${ }^{\text {a }}$, B. Liverani ${ }^{\text {a }}$, M. Taviani ${ }^{b}$ \\ a Department of Biological Ceological and Environmental Sciences, University of Bologna, Via Zamboni 67, 40126 Bologna, Itaby \\ bInstitute of Marine Sciences (ISMAR)-CNR, Via Gobetti 101, 40129 Bologna, Italy \\ 'Department of Earth and Environmental Sciences, BIUST, Private Bag 16, Palapye, Botswana
}

\section{A R T I C L E I N F O}

Keywords:

Carbonate chimneys

Hydrocarbon migration

Avaerobic methane oxidation

Apennine chain

\begin{abstract}
A B S T R A C T
A remarkable exposure of carbonate pipe like and slab concretions occurs along the Enza riverbanks on the Northern Apennines foothills (Italy). Based upon geochemical and field evidences, their genesis has been attributed to microbial governed carbonate precipitation from hydrocarbon enriched fluids. The pipe like concretions are thus interpreted as former conduits (chimneys) marking sites of methane ascent onto the seafloor. The resulting Enza River chimney field is arranged in a palisade fashion, a rare example of such. Mineralogical and petrographic analyses of some chimneys and slabs document that the dominant cement is dolomite. Although the chimneys show a rather homogeneous texture, a clear zonation is observed in the relative distribution of major and minor elements in their internal and external parts. The occurrence of sulphide minerals in the stratigraphically upper samples indicates possible renewal of fluids leakage after a major erosive event.
\end{abstract}

\section{Introduction}

The formation of authigenic carbonates linked to hydrocarbon enriched fluids seepage onto the seafloor is a well known phenomenon. It is documented in modern and ancient marine sedimentary basins worldwide particularly, but not exclusively, in compressive tectonic regimes at convergent margins (e.g., Taviani, 2001; Pinheiro et al., 2003; Nyman et al., 2010; Capozzi et al., 2012). Microbially mediated anaerobic oxidation of methane (AOM) and sulphate reduction (SR) increase the pore water alkalinity, favouring the pre cipitation of methane derived authigenic carbonates (MDAC) (e.g. Boetius et al., 2000; Peckmann and Thiel, 2004; Teichert et al., 2005). Their various morphologies include slabs and crusts, cylindrical and pipe like conduits, and irregularly shaped bodies (e.g. Taviani, 2001; Clari et al., 2004; Lein, 2004; Magalhaes et al., 2012). Pipe like con duits are the less reported in literature and their formation mecha nisms are still under debate (e.g. Nyman et al., 2010; Magalhaes et al., 2012; Oppo et al., in this issue).

Increasing attention is presently devoted to understanding pipelike concretions characteristics and formative processes (e.g., Orpin, 1997; Diaz del Rio et al., 2001, 2003; Somoza et al., 2003; De Boever et al., 2006a,b; Nyman et al., 2010; Magalhaes et al., 2012;

\footnotetext{
* Corresponding author.

E mail address: irene.viola3@unibo.it (I. Viola).
}

Oppo et al., in this issue; Angeletti et al., in this issue). The seep carbonates show characteristic mineralogy and geochemistry, as the authigenic carbonate minerals, such as dolomite, calcite and aragonite, which compose the MDAC concretions, are usually over than $50 \%$ bulk weight. Their relative amounts depend on the formation environment and on the fluids involved in the precipitation (e.g. Peckmann and Thiel, 2004; Magalhaes et al., 2012; Vanneste et al., 2012). Being considered as indicators of hydrocarbon seepages, authigenic carbonates testify the present day or past occurrence of petroleum generation systems (Capozzi et al., 2012; Oppo et al., 2013; Unterseh, 2013).

This study provides first detailed mineralogical, geochemical and petrographic information on hydrocarbon imprinted carbonates, from the Enza River succession (Capozzi et al., 2013; Gunderson et al., 2014; Oppo et al., in this issue). In addition, we also provide some compositional data on the host marine sediments for a better characterization of the genetic environments of carbonates. At this site, the formation of methane derived authigenic carbonates is strictly connected with the geologic evolution of the basin (see Oppo et al., in this issue).

\section{Geological setting}

Intense flooding events over the last few years resulted in a pronounced fluvial incision of the Enza riverbanks in the Northern Apennines foothills (Fig. 1). 
Such erosive processes resulted in the exposure downstream the city of San Polo d'Enza of a marine succession punctuated by a number of slab and pipe like carbonate concretions (Fig. 2).

The foothills deformation in the Enza River area started in the Late Miocene and continued until the Pleistocene, resulting in the formation of the Quattro Castella anticline (Boccaletti et al., 2010; Ponza et al., 2010; Gunderson et al., 2014). The Enza River succession belongs to the forelimb of the Quattro Castella anticline and represents a full transition, from muddy shelfal deposits (Argille Azzurre Fm.) to the transitional coastal system (Sabbie Gialle Fm.) (Gunderson et al., 2014, and references therein). The synsedimentary tilting of the anticline led to the variation of strata dip along the $65 \mathrm{~m}$ thick studied succession, which progressively changes from $55^{\circ}$ at the base to $42^{\circ}$ at the top. A corallinaceous rhodolith rich bed, which dips $35^{\circ}$, represents the top of the studied section and identifies an angular unconformity (Gunderson et al., 2014) that marks an erosional discontinuity. The recovering of deposition started with the colonization by benthic epifauna (Oppo et al., in this issue).

The carbonate concretions are enclosed in the Lower Pleistocene Argille Azzurre Fm., which is composed of blue grey silty clays deposited in external continental shelf environment. The carbonate pipe like concretions, in this paper defined as chimneys, and slabs occur between 30 and $65 \mathrm{~m}$ of the Enza section (Oppo et al., in this issue). The chimneys have often sub vertical orientation with respect to horizontal and are arranged in a sort of pipe palisade, a rare occurrence seldom seen in outcrop (e.g. De Boever et al., 2006a; Cau et al., in this issue) and submerged situations (e.g. Taviani, 2014) whilst slabs conform to stratification. Morphologies and dimensions vary: pipe like and cylindrical chimneys range from few $\mathrm{cm}$ to almost $2 \mathrm{~m}$ high, with up to $40 \mathrm{~cm}$ in diameter; slabs are few $\mathrm{cm}$ to $30 \mathrm{~cm}$ thick and up to several metres long (Fig. 2) (see also Capozzi et al., 2013; Gunderson et al., 2014; Oppo et al., in this issue).

\section{Materials and methods}

Fieldwork was conducted in May 2010 and May 2013 to sample chimneys, slabs and host sediment for their mineralogical, geochemical and petrographical comparative characterization. 31 samples of Argille Azzurre Fm. have been recovered and analysed, together with 4 samples of carbonate concretions: two chimneys (En5 and En10) and two carbonate bodies, one tabular slab $(\mathrm{Cr} 2)$ and a concretion within the rhodolith rich layer (Cr3) (Fig. 3).

The carbonate concretions have been divided in 29 sub samples to be analysed (Fig. 4).

The En5 chimney does not have an open central vent and is $63 \mathrm{~cm}$ long and $20 \mathrm{~cm}$ in diameter (Fig. 4a). To investigate eventual geochemical variations along the chimney, it has been cut in 8 sections from the bottom (1) to the top (8). Each section, excluding 1 and 8 , has been longitudinally divided in 3 subsamples: internal part (A), central part (B) and external part (C). The En10 chimney is

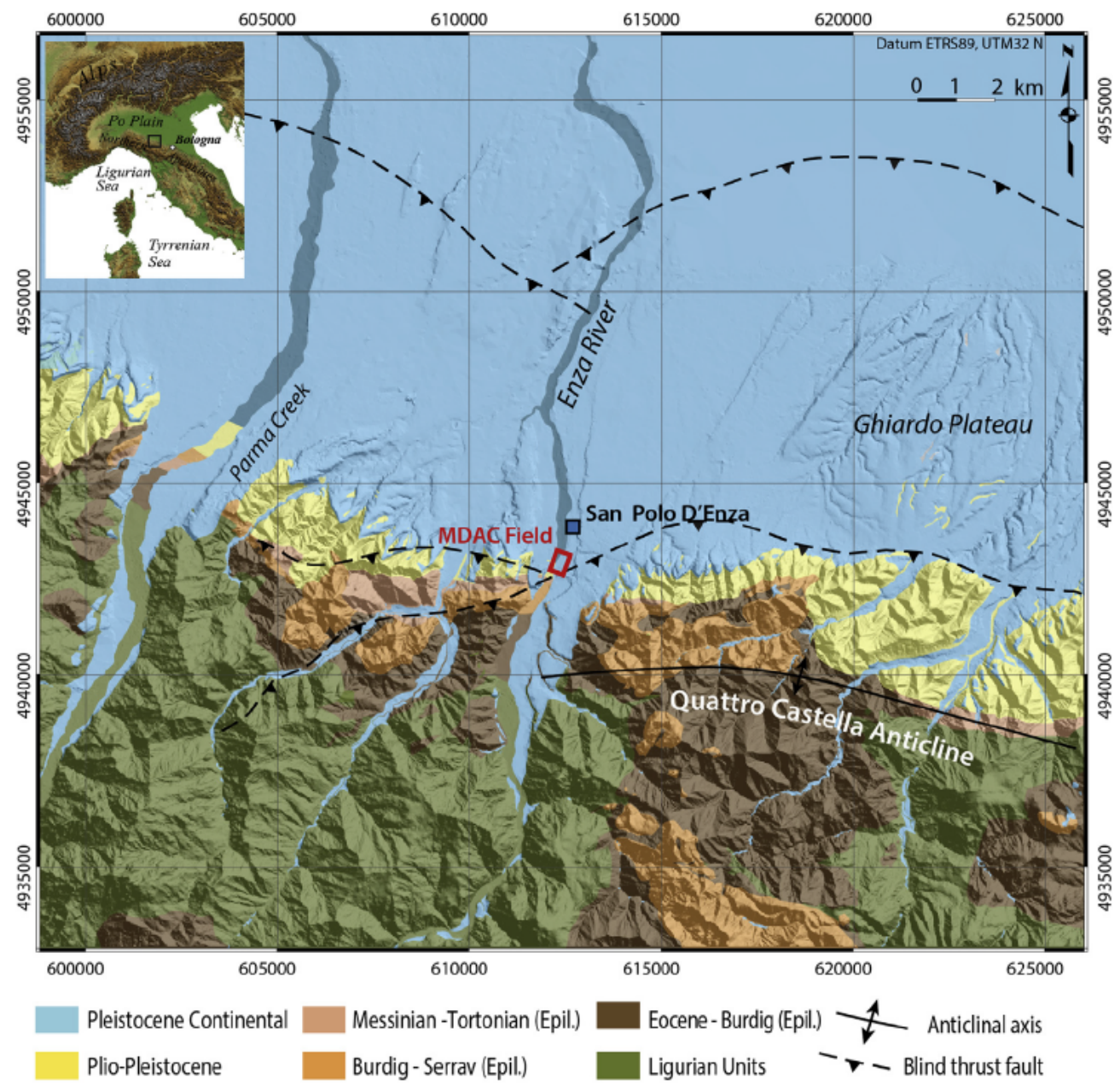

Figure 1. Geological map of the investigated area of the Northern Apennines. The Enza carbonate field (red square) is located along the Enza River near the town of San Polo d'Enza (modified from Oppo et al., in this issue). (For interpretation of the references to colour in this figure legend, the reader is referred to the web version of this article.) 


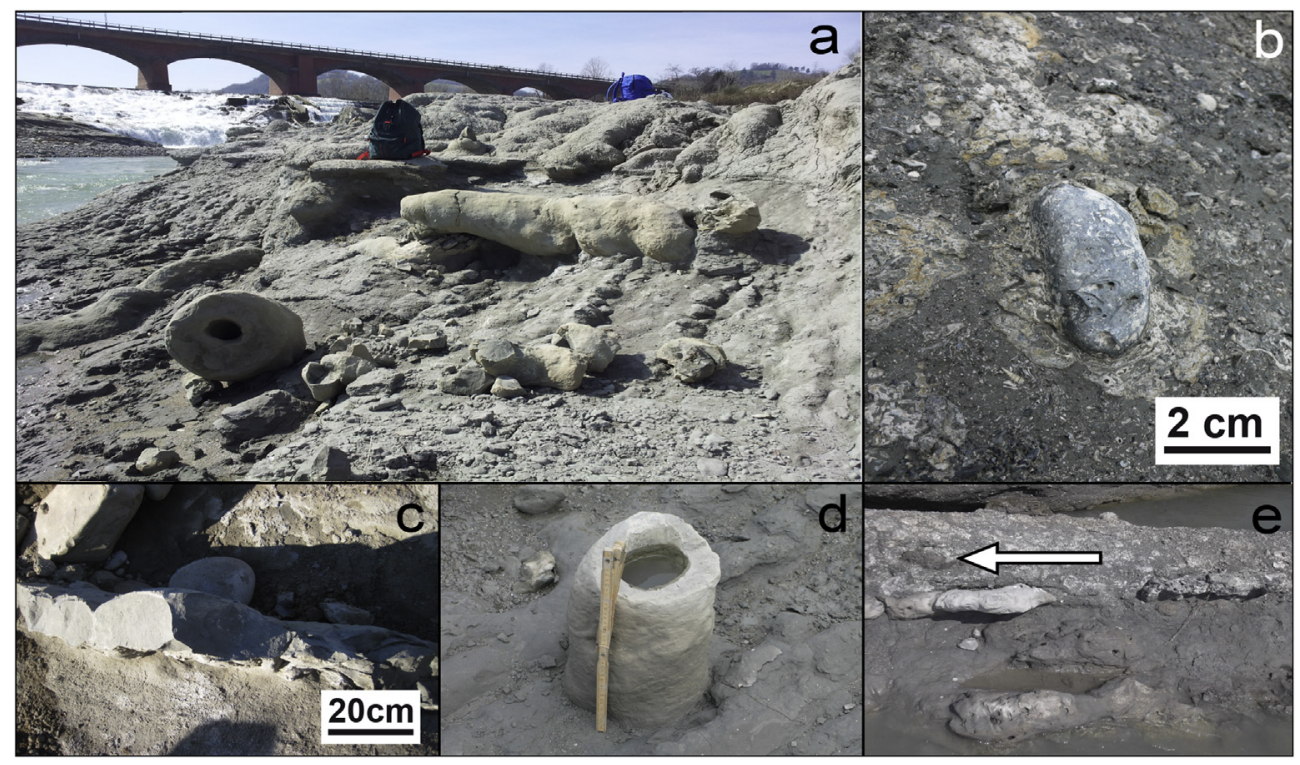

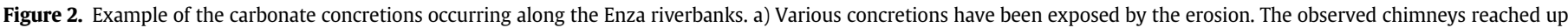

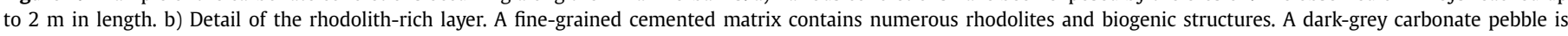

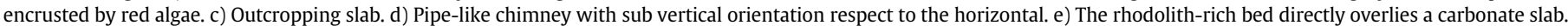
Arrow points to dark-grey carbonate enclosed in the bed.

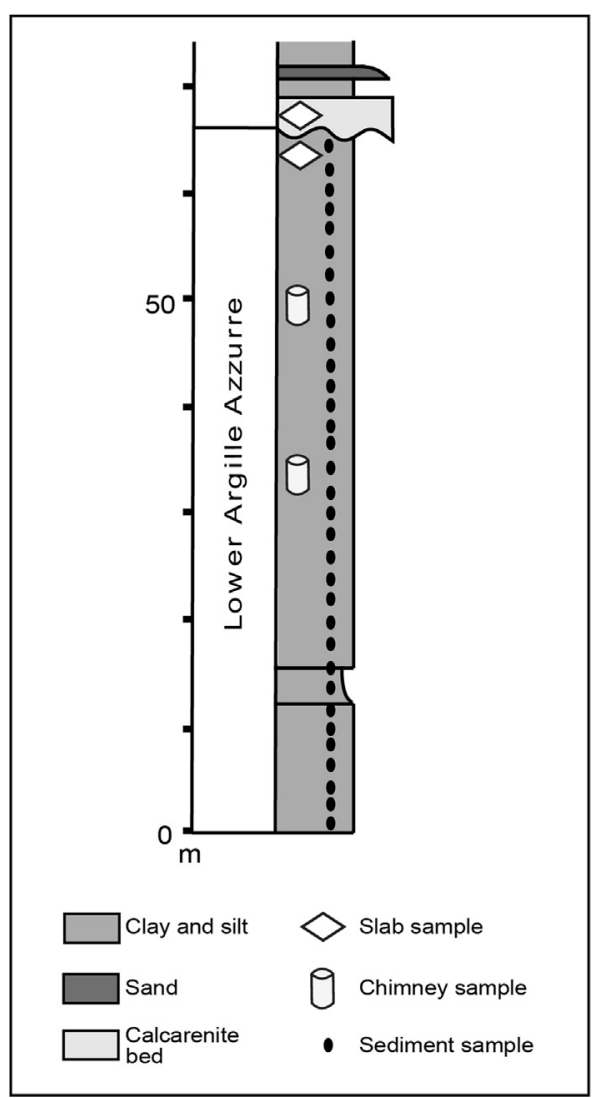

Figure 3. Simplified stratigraphic column of the studied succession along the Enza River. The rhodolith-rich layer marks an erosional unconformity, separating the interval hosting the concretions from the underlying Lower Pleistocene succession. The position of samples discussed in text is marked. Sediments are sampled from 4 to $64 \mathrm{~m}$ bottom top; chimney En5 is the lower one, En10 the upper one; slab $\mathrm{Cr} 2$ is the one just below the corallinaceus bed, while $\mathrm{Cr} 3$ is the one collected inside the corallinaceus bed.
$170 \mathrm{~cm}$ long and $20 \mathrm{~cm}$ in diameter; only the top and the bottom have been analysed (Fig. $4 \mathrm{~b}$ and c, respectively). The top subsample has been divided in external (B) and internal (A) portions.

The $\mathrm{Cr} 3$ sample is a concretion within the rhodolith rich layer; it shows a dark colour and is well cemented. A dark grey area $(\mathrm{Cr} 3 \mathrm{~A}$, Fig. 4d) was analysed and compared with the light grey matrix (Cr3B, Fig. 4e). The $\mathrm{Cr} 2$ slab pertained to the carbonate concretions stratigraphically below the rhodolith rich layer; it shows a grey colour with a light grey area (Cr2b in Fig. 4f).

The samples have been powdered, homogenized in agate mortar and analysed for mineralogy by X ray diffractometry (XRD) using a Philips PW 1130 ( $\mathrm{Cu} \mathrm{K \alpha}$ radiation Ni filtered) in the XRD Laboratory of BiGeA department, Bologna University. Powders were pressed into alumina holders in order to avoid preferential orientation of sheet silicates. Estimates of the relative minerals abundance were determined using MacDiff software packages and carbonate min eral compositional limits defined according to Goldsmith and Graf (1958) and Lumsden (1979).

Nitrogen and Total Organic Carbon (TOC) have been analysed with CHNS pre treating $10 \mathrm{mg}$ of powdered sample in $40 \mu \mathrm{l}$ of $\mathrm{HCl}$ $1.5 \mathrm{~N}$ for $2 \mathrm{~h}$ in the oven, this procedure have been repeated two times to eliminate all the inorganic carbon traces. The resulting compounds have been analysed to estimate TOC and Nitrogen by CHNS Analizer Flash 2000 Thermo Scientific Gas Chromatographer in Pedology Laboratory, BiGeA department, Ravenna, Bologna University. This analysis was performed only on En5 samples and hosting sediments because the higher number of samples from En5 gives a better representation of the carbonates bodies in all its parts (internal, central and external) and were more accurate for a comparison with the hosting sediments samples.

Major and trace elements have been determined by $\mathrm{X}$ ray fluorescence (XRF) spectrometry on pressed powered pellets using a Philips PW 1480 automated spectrometer following the methods of Franzini et al. (1972, 1975), Leoni and Saitta (1976) and Leoni et al.(1982) for matrix corrections in XRF Laboratory, BiGeA department, Bologna University. The estimated precision and accuracy for trace element determinations are more than 5\% except for the elements at $10 \mathrm{ppm}$ and lower (10-15\%); the detection limit for most of the trace elements is $3 \mathrm{ppm}$ (Leoni and Saitta, 1976). 


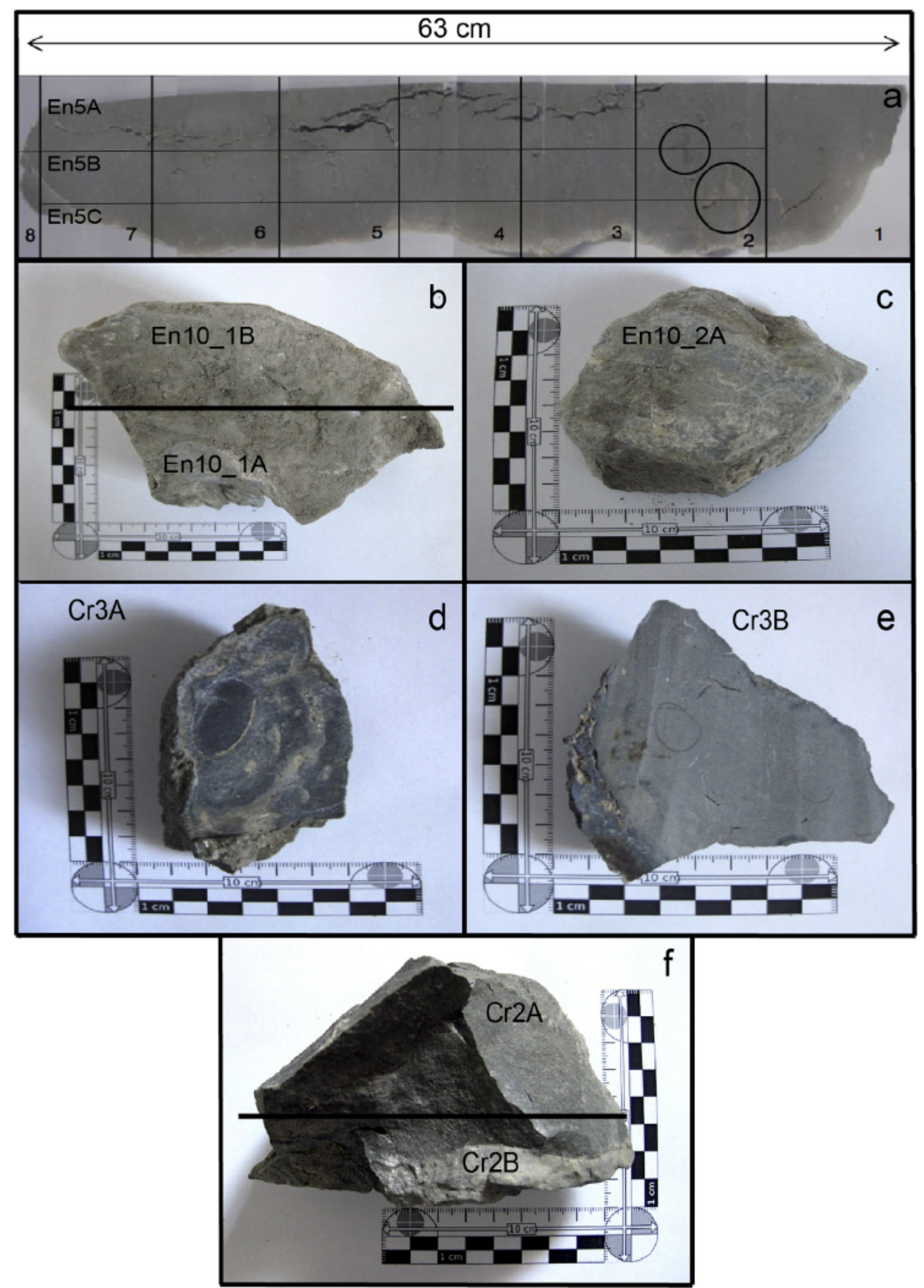

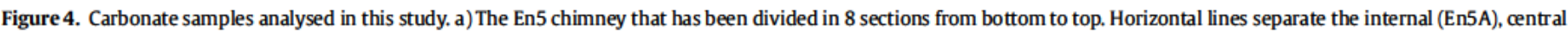

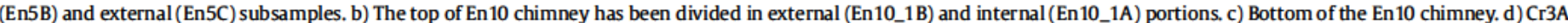

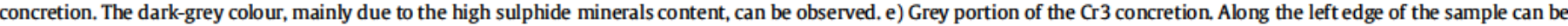

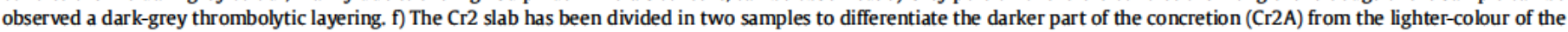
external area (Cr2B). (For interpretation of the references to colour in this figure legend, the reader is referred to the web version of this article.)

The petrographic and microfacies based analyses were performed on. 80 uncovered thin sections $\left(45 \times 60 \mathrm{~mm}^{2}\right.$ surface area, $30 \mu \mathrm{m}$ thickness) at the ISMAR CNR, U.O.S. Bologna. Cath odoluminescence petrography was conducted using a CL $8200 \mathrm{MK} 3$ optical equipment (operating conditions: $20 \mathrm{kV}$ beam voltage and $200 \mu \AA ̊$ beam current) at the University of Padua. Morphological description and chemical elemental composition of microscopic features were obtained by using a Philips 515 scanning electron microscope (SEM) equipped with an electron back scattering sys tem and EDX (EDAX DX 4) at the SEM Laboratory, BiGeA department University of Bologna. SEM EDX investigations (SEM operating conditions: 15 and $25 \mathrm{kV}$ accelerating voltage) were performed on freshly broken samples (Au coated) and thin sections (C coated).

Carbon and oxygen isotopic analyses have been performed on carbonate samples from Enza River in this study at CNR Institute 
Table 1

Mineral abundances in the sediment. Samples position starts from the base of the Enza section.

\begin{tabular}{|c|c|c|c|c|c|c|c|c|c|c|}
\hline \multirow[t]{2}{*}{ Samples } & Position & Quartz & Chlorite & Muscovite & Calcite & Dolomite & Feldspars & Pladgioclase & Clay minerals & Others \\
\hline & $(\mathrm{m})$ & $(\%)$ & $(\%)$ & $(\%)$ & $(\%)$ & $(\%)$ & $(\%)$ & (\%) & $(\%)$ & $(\%)$ \\
\hline 4 & 1.2 & 20 & 6 & 9 & 21 & 2 & 15 & 10 & 15 & 2 \\
\hline 12 & 4.4 & 27 & 7 & 9 & 18 & 0 & 13 & 6 & 19 & 1 \\
\hline 17 & 6.4 & 23 & 9 & 8 & 19 & 0 & 11 & 10 & 17 & 3 \\
\hline 22 & 8.4 & 16 & 6 & 6 & 28 & 2 & 7 & 11 & 23 & 1 \\
\hline 27 & 10.4 & 11 & 5 & 8 & 31 & 0 & 6 & 14 & 23 & 2 \\
\hline 32 & 12.4 & 24 & 8 & 10 & 19 & 0 & 9 & 15 & 11 & 4 \\
\hline 37 & 14.4 & 15 & 7 & 8 & 25 & 0 & 8 & 5 & 30 & 2 \\
\hline 42 & 16.4 & 21 & 9 & 10 & 20 & 0 & 11 & 6 & 20 & 3 \\
\hline 47 & 18.4 & 18 & 7 & 7 & 22 & 2 & 13 & 11 & 16 & 4 \\
\hline 52 & 20.4 & 17 & 5 & 6 & 28 & 2 & 8 & 9 & 23 & 2 \\
\hline 57 & 22.4 & 24 & 8 & 8 & 17 & 0 & 17 & 4 & 19 & 3 \\
\hline 62 & 24.4 & 16 & 5 & 7 & 29 & 0 & 8 & 6 & 25 & 4 \\
\hline 67 & 26.4 & 26 & 6 & 9 & 19 & 0 & 12 & 11 & 15 & 2 \\
\hline 72 & 28.4 & 17 & 7 & 6 & 26 & 1 & 9 & 13 & 19 & 2 \\
\hline 77 & 30.4 & 23 & 6 & 7 & 21 & 3 & 12 & 10 & 15 & 3 \\
\hline 82 & 32.4 & 18 & 7 & 7 & 22 & 3 & 13 & 8 & 16 & 6 \\
\hline 88 & 34.8 & 25 & 7 & 10 & 14 & 0 & 20 & 12 & 11 & 1 \\
\hline 92 & 36.4 & 24 & 9 & 11 & 15 & 4 & 9 & 8 & 17 & 3 \\
\hline 97 & 38.4 & 21 & 6 & 8 & 15 & 2 & 24 & 7 & 15 & 2 \\
\hline 102 & 40.4 & 13 & 10 & 7 & 23 & 2 & 9 & 10 & 24 & 2 \\
\hline 107 & 42.4 & 21 & 8 & 8 & 20 & 3 & 9 & 9 & 19 & 3 \\
\hline 113 & 44.8 & 12 & 5 & 5 & 31 & 2 & 8 & 14 & 21 & 2 \\
\hline 117 & 46.4 & 25 & 7 & 10 & 17 & 2 & 13 & 12 & 10 & 4 \\
\hline 122 & 48.4 & 14 & 7 & 7 & 25 & 1 & 10 & 4 & 29 & 3 \\
\hline 127 & 50.4 & 25 & 7 & 7 & 20 & 2 & 12 & 9 & 17 & 1 \\
\hline 132 & 52.4 & 13 & 11 & 6 & 23 & 2 & 10 & 12 & 20 & 3 \\
\hline 137 & 54.4 & 20 & 8 & 9 & 21 & 0 & 12 & 10 & 19 & 1 \\
\hline 142 & 56.4 & 25 & 5 & 8 & 17 & 2 & 17 & 8 & 16 & 2 \\
\hline 147 & 58.4 & 32 & 10 & 9 & 13 & 0 & 14 & 6 & 12 & 4 \\
\hline 152 & 60.4 & 16 & 4 & 4 & 30 & 1 & 9 & 14 & 19 & 3 \\
\hline 157 & 62.4 & 24 & 8 & 7 & 18 & 2 & 17 & 7 & 15 & 2 \\
\hline 162 & 64.4 & 21 & 6 & 9 & 20 & 2 & 12 & 7 & 21 & 2 \\
\hline
\end{tabular}

Table 2

Major oxides in the sediment. Samples position starts from the base of the Enza section.

\begin{tabular}{|c|c|c|c|c|c|c|c|c|c|c|c|c|c|c|}
\hline \multirow[t]{2}{*}{ Sample } & \multirow{2}{*}{$\frac{\text { Position }}{(\mathrm{m})}$} & \multirow{2}{*}{$\frac{\mathrm{SiO}_{2}}{\text { (wt \%) }}$} & \multirow{2}{*}{$\frac{\mathrm{TiO}_{2}}{\text { (wt \%) }}$} & \multirow{2}{*}{$\frac{\mathrm{Al}_{2} \mathrm{O}_{3}}{(\mathrm{wt} \%)}$} & \multirow{2}{*}{$\frac{\mathrm{Fe}_{2} \mathrm{O}_{3}}{\text { (wt \%) }}$} & \multirow{2}{*}{$\frac{\mathrm{MnO}}{\text { (wt \%) }}$} & \multirow{2}{*}{$\frac{\mathrm{MgO}}{(\mathrm{wt} \%)}$} & \multirow{2}{*}{$\frac{\mathrm{CaO}}{(\mathrm{wt} \%)}$} & \multirow{2}{*}{$\frac{\mathrm{Na}_{2} \mathrm{O}}{(\mathrm{wt} \%)}$} & \multirow{2}{*}{$\frac{\mathrm{K}_{2} \mathrm{O}}{\text { (wt \%) }}$} & \multirow{2}{*}{$\frac{\mathrm{P}_{2} \mathrm{O}_{5}}{\text { (wt \%) }}$} & \multirow{2}{*}{$\frac{\text { LOI }}{(w t \%)}$} & \multirow[t]{2}{*}{$\mathrm{Mg} / \mathrm{Ca}$} & \multirow[t]{2}{*}{$\mathrm{Sr} / \mathrm{Ca}$} \\
\hline & & & & & & & & & & & & & & \\
\hline 4 & 1.20 & 44.70 & 0.60 & 12.25 & 5.20 & 0.12 & 3.31 & 15.28 & 0.98 & 2.11 & 0.11 & 15.36 & 0.1828 & 0.0041 \\
\hline 12 & 4.40 & 45.93 & 0.58 & 12.67 & 4.83 & 0.12 & 3.41 & 14.42 & 1.04 & 2.16 & 0.07 & 14.78 & 0.1996 & 0.0042 \\
\hline 17 & 6.40 & 45.29 & 0.58 & 12.25 & 4.90 & 0.12 & 3.39 & 15.50 & 1.07 & 2.12 & 0.06 & 14.70 & 0.1846 & 0.0043 \\
\hline 22 & 8.40 & 40.64 & 0.56 & 11.88 & 4.63 & 0.11 & 2.94 & 17.90 & 0.98 & 1.96 & 0.05 & 18.34 & 0.1386 & 0.0041 \\
\hline 27 & 10.40 & 40.55 & 0.57 & 12.34 & 4.81 & 0.11 & 2.77 & 16.72 & 0.93 & 2.15 & 0.08 & 18.97 & 0.1398 & 0.0048 \\
\hline 32 & 12.40 & 45.94 & 0.58 & 12.84 & 5.02 & 0.12 & 3.31 & 14.06 & 1.12 & 2.13 & 0.07 & 14.81 & 0.1987 & 0.0046 \\
\hline 37 & 14.40 & 45.99 & 0.56 & 12.45 & 4.57 & 0.12 & 3.17 & 15.00 & 1.05 & 2.07 & 0.07 & 14.95 & 0.1783 & 0.0049 \\
\hline 42 & 16.40 & 46.12 & 0.57 & 12.60 & 4.64 & 0.12 & 3.35 & 14.16 & 0.95 & 2.11 & 0.07 & 15.30 & 0.1996 & 0.0037 \\
\hline 47 & 18.40 & 46.57 & 0.58 & 12.80 & 4.59 & 0.11 & 3.28 & 13.95 & 0.97 & 2.14 & 0.07 & 14.93 & 0.1984 & 0.0044 \\
\hline 52 & 20.40 & 45.54 & 0.58 & 12.92 & 4.76 & 0.11 & 3.36 & 14.29 & 1.00 & 2.15 & 0.07 & 15.22 & 0.1984 & 0.0036 \\
\hline 57 & 22.40 & 45.88 & 0.58 & 12.85 & 4.92 & 0.12 & 3.39 & 14.60 & 0.98 & 2.14 & 0.12 & 14.43 & 0.1959 & 0.0040 \\
\hline 62 & 24.40 & 46.64 & 0.54 & 12.07 & 4.09 & 0.12 & 3.45 & 15.08 & 1.01 & 1.96 & 0.08 & 14.95 & 0.1931 & 0.0029 \\
\hline 67 & 26.40 & 47.06 & 0.57 & 12.65 & 4.77 & 0.12 & 3.43 & 13.99 & 1.06 & 2.15 & 0.05 & 14.16 & 0.2069 & 0.0041 \\
\hline 72 & 28.40 & 46.45 & 0.58 & 13.19 & 4.75 & 0.11 & 3.51 & 13.46 & 0.96 & 2.22 & 0.09 & 14.69 & 0.2201 & 0.0040 \\
\hline 77 & 30.40 & 45.97 & 0.58 & 12.60 & 4.63 & 0.11 & 3.68 & 14.32 & 1.00 & 2.12 & 0.06 & 14.92 & 0.2169 & 0.0037 \\
\hline 82 & 32.40 & 45.20 & 0.60 & 12.77 & 4.77 & 0.11 & 3.75 & 14.69 & 1.01 & 2.14 & 0.07 & 14.88 & 0.2154 & 0.0046 \\
\hline 88 & 34.80 & 48.54 & 0.62 & 13.67 & 4.87 & 0.11 & 3.71 & 12.11 & 1.05 & 2.26 & 0.10 & 12.96 & 0.2585 & 0.0043 \\
\hline 92 & 36.40 & 48.06 & 0.57 & 12.59 & 4.54 & 0.11 & 3.55 & 13.65 & 1.08 & 2.11 & 0.07 & 13.68 & 0.2195 & 0.0042 \\
\hline 97 & 38.40 & 48.36 & 0.60 & 13.31 & 4.99 & 0.11 & 3.50 & 12.41 & 1.02 & 2.25 & 0.10 & 13.34 & 0.2380 & 0.0041 \\
\hline 102 & 40.40 & 46.55 & 0.62 & 13.40 & 5.13 & 0.11 & 3.46 & 12.97 & 1.00 & 2.31 & 0.12 & 14.33 & 0.2251 & 0.0044 \\
\hline 107 & 42.40 & 46.05 & 0.60 & 13.13 & 4.87 & 0.12 & 3.47 & 13.62 & 1.01 & 2.22 & 0.09 & 14.82 & 0.2150 & 0.0044 \\
\hline 113 & 44.80 & 47.17 & 0.57 & 12.88 & 4.70 & 0.12 & 3.56 & 14.05 & 1.10 & 2.08 & 0.06 & 13.72 & 0.2138 & 0.0040 \\
\hline 117 & 46.40 & 47.44 & 0.59 & 13.13 & 4.77 & 0.11 & 3.42 & 13.20 & 1.03 & 2.19 & 0.11 & 14.00 & 0.2186 & 0.0043 \\
\hline 122 & 48.40 & 48.12 & 0.63 & 14.28 & 5.21 & 0.11 & 3.37 & 11.37 & 1.00 & 2.35 & 0.08 & 13.49 & 0.2501 & 0.0039 \\
\hline 127 & 50.40 & 47.73 & 0.61 & 12.51 & 4.77 & 0.11 & 3.54 & 13.77 & 1.10 & 2.20 & 0.08 & 13.57 & 0.2169 & 0.0042 \\
\hline 132 & 52.40 & 47.93 & 0.59 & 12.36 & 4.85 & 0.12 & 3.54 & 13.59 & 1.14 & 2.10 & 0.11 & 13.68 & 0.2198 & 0.0038 \\
\hline 137 & 54.40 & 47.93 & 0.59 & 12.91 & 4.65 & 0.11 & 3.33 & 13.29 & 1.06 & 2.12 & 0.08 & 13.93 & 0.2114 & 0.0040 \\
\hline 142 & 56.40 & 48.28 & 0.56 & 12.34 & 4.57 & 0.13 & 3.49 & 14.28 & 1.27 & 2.01 & 0.13 & 12.93 & 0.2062 & 0.0037 \\
\hline 147 & 58.40 & 46.38 & 0.59 & 12.58 & 4.73 & 0.12 & 3.37 & 14.43 & 0.89 & 2.09 & 0.09 & 14.74 & 0.1971 & 0.0036 \\
\hline 152 & 60.40 & 48.07 & 0.58 & 12.56 & 4.54 & 0.11 & 3.28 & 13.79 & 1.00 & 2.05 & 0.09 & 13.93 & 0.2007 & 0.0038 \\
\hline 157 & 62.40 & 49.09 & 0.54 & 12.05 & 4.30 & 0.12 & 3.32 & 14.46 & 1.24 & 1.94 & 0.09 & 13.25 & 0.1937 & 0.0041 \\
\hline 162 & 64.40 & 50.63 & 0.55 & 12.50 & 4.32 & 0.11 & 3.29 & 13.19 & 1.17 & 1.97 & 0.10 & 12.17 & 0.2105 & 0.0038 \\
\hline
\end{tabular}


Table 3

Minor elements in the sediment. Samples position starts from the base of the Enza section.

\begin{tabular}{|c|c|c|c|c|c|c|c|c|c|c|c|c|c|c|c|c|c|c|c|c|c|c|c|}
\hline Sample & Position & Sc & V & $\mathrm{Cr}$ & Co & $\mathrm{Ni}$ & $\mathrm{Cu}$ & $\mathrm{Zn}$ & As & $\mathrm{Rb}$ & $\mathrm{Sr}$ & Y & $\mathrm{Zr}$ & $\mathrm{Nb}$ & $\mathrm{Ba}$ & La & $\mathrm{Ce}$ & $\mathrm{Pb}$ & Th & $\mathrm{S}$ & $\mathrm{Br}$ & Mo & TOC \\
\hline & (m) & (ppm) & (ppm) & $(\mathrm{ppm})$ & $(\mathrm{ppm})$ & (ppm) & $(\mathrm{ppm})$ & $(\mathrm{ppm})$ & $(\mathrm{ppm})$ & (ppm) & $(\mathrm{ppm})$ & $(\mathrm{ppm})$ & (ppm) & (ppm) & $(\mathrm{ppm})$ & $(\mathrm{ppm})$ & $(\mathrm{ppm})$ & (ppm) & (ppm) & $(\mathrm{ppm})$ & (ppm) & $(\mathrm{ppm})$ & (\%) \\
\hline 4 & 1.2 & 9 & 94 & 138 & 16 & 80 & 19 & 74 & 7 & 113 & 451 & 19 & 110 & 10 & 281 & 15 & 61 & 14 & 2 & 2250 & $<20$ & $<3$ & 0.56 \\
\hline 12 & 4.4 & 11 & 101 & 143 & 14 & 88 & 35 & 93 & 7 & 114 & 429 & 18 & 96 & 10 & 287 & 22 & 67 & 6 & 2 & 770 & $<20$ & $<3$ & 0.52 \\
\hline 17 & 6.4 & 7 & 98 & 134 & 11 & 80 & 18 & 70 & 10 & 114 & 473 & 20 & 105 & 11 & 313 & 35 & 62 & 3 & 9 & 1910 & $<20$ & 3 & 0.5 \\
\hline 22 & 8.4 & 2 & 100 & 114 & 12 & 74 & 23 & 95 & 5 & 103 & 531 & 16 & 67 & 10 & 261 & 38 & 69 & 4 & 12 & 1430 & $<20$ & $<3$ & 0.68 \\
\hline 27 & 10.4 & 10 & 104 & 108 & 11 & 74 & 29 & 103 & 10 & 131 & 578 & 22 & 53 & 13 & 293 & 26 & 62 & 11 & 12 & 750 & $<20$ & 3 & 0.69 \\
\hline 32 & 12.4 & 11 & 94 & 136 & 16 & 80 & 22 & 96 & 5 & 118 & 464 & 22 & 128 & 13 & 286 & 24 & 62 & 8 & 10 & 670 & $<20$ & $<3$ & 0.46 \\
\hline 37 & 14.4 & 7 & 95 & 132 & 13 & 77 & 33 & 94 & 14 & 131 & 522 & 24 & 106 & 13 & 318 & 22 & 50 & 7 & 7 & 2890 & $<20$ & $<3$ & 0.57 \\
\hline 42 & 16.4 & 9 & 100 & 124 & 11 & 80 & 22 & 94 & $<3$ & 104 & 379 & 17 & 88 & 8 & 266 & 13 & 58 & 7 & 10 & 1600 & $<20$ & $<3$ & 0.67 \\
\hline 47 & 18.4 & 9 & 98 & 128 & 10 & 79 & 23 & 101 & 11 & 120 & 435 & 21 & 101 & 9 & 282 & 21 & 60 & 9 & 15 & 2990 & $<20$ & $<3$ & 0.58 \\
\hline 52 & 20.4 & 4 & 101 & 138 & 11 & 85 & 25 & 100 & $<3$ & 103 & 366 & 14 & 84 & 10 & 290 & 22 & 75 & 9 & 12 & 610 & $<20$ & 3 & 0.62 \\
\hline 57 & 22.4 & 12 & 104 & 136 & 16 & 87 & 34 & 98 & 20 & 111 & 419 & 20 & 79 & 9 & 280 & 31 & 60 & 7 & 14 & 3310 & $<20$ & 3 & 0.61 \\
\hline 62 & 24.4 & 16 & 87 & 134 & 14 & 74 & 23 & 82 & 11 & 71 & 317 & 14 & 67 & 9 & 251 & 26 & 56 & 2 & 11 & 1670 & $<20$ & $<3$ & 0.5 \\
\hline 67 & 26.4 & 13 & 102 & 147 & 11 & 87 & 19 & 93 & 21 & 111 & 415 & 18 & 102 & 10 & 280 & 19 & 58 & 2 & 7 & 2800 & $<20$ & $<3$ & 0.46 \\
\hline 72 & 28.4 & 10 & 106 & 141 & 15 & 98 & 23 & 96 & 2 & 115 & 385 & 19 & 93 & 11 & 275 & 25 & 50 & 7 & 12 & 2580 & $<20$ & $<3$ & 0.44 \\
\hline 77 & 30.4 & 7 & 104 & 158 & 13 & 96 & 22 & 99 & 9 & 99 & 374 & 18 & 88 & 12 & 274 & 28 & 69 & 7 & 6 & 2600 & $<20$ & $<3$ & 0.38 \\
\hline 82 & 32.4 & 12 & 106 & 165 & 13 & 88 & 16 & 97 & 13 & 122 & 488 & 23 & 122 & 12 & 283 & 20 & 68 & 10 & 9 & 2830 & $<20$ & $<3$ & 0.42 \\
\hline 88 & 34.8 & 12 & 105 & 166 & 13 & 87 & 19 & 95 & 6 & 114 & 371 & 23 & 165 & 11 & 280 & 29 & 87 & 8 & 9 & 1590 & $<20$ & $<3$ & 0.35 \\
\hline 92 & 36.4 & 13 & 102 & 139 & 10 & 76 & 17 & 88 & $<3$ & 111 & 405 & 23 & 141 & 8 & 297 & 13 & 61 & 11 & 14 & 1980 & $<20$ & 3 & 0.41 \\
\hline 97 & 38.4 & 14 & 109 & 148 & 14 & 85 & 32 & 96 & 5 & 109 & 360 & 22 & 118 & 10 & 318 & 21 & 59 & 4 & 16 & 2760 & $<20$ & $<3$ & 0.41 \\
\hline 102 & 40.4 & 11 & 106 & 126 & 11 & 76 & 17 & 82 & $<3$ & 129 & 407 & 21 & 104 & 13 & 286 & 28 & 81 & 9 & 16 & 1750 & $<20$ & 3 & 0.44 \\
\hline 107 & 42.4 & 11 & 111 & 142 & 13 & 77 & 24 & 101 & 11 & 118 & 429 & 20 & 102 & 10 & 318 & 29 & 73 & 8 & 12 & 1590 & $<20$ & $<3$ & 0.43 \\
\hline 113 & 44.8 & 8 & 101 & 132 & 13 & 71 & 19 & 88 & 16 & 111 & 403 & 21 & 143 & 11 & 289 & 17 & 82 & 10 & 7 & 2260 & $<20$ & $<3$ & 0.39 \\
\hline 117 & 46.4 & 13 & 101 & 132 & 10 & 74 & 17 & 74 & 4 & 122 & 403 & 20 & 129 & 12 & 263 & 29 & 72 & 5 & 10 & 1600 & $<20$ & $<3$ & 0.43 \\
\hline 122 & 48.4 & 13 & 113 & 158 & 12 & 87 & 19 & 101 & 6 & 108 & 313 & 18 & 94 & 10 & 294 & 28 & 75 & 13 & 10 & 2860 & $<20$ & $<3$ & 0.42 \\
\hline 127 & 50.4 & 8 & 100 & 137 & 13 & 79 & 14 & 100 & 9 & 116 & 414 & 26 & 136 & 13 & 321 & 15 & 46 & 11 & 6 & 3660 & $<20$ & $<3$ & 0.39 \\
\hline 132 & 52.4 & 16 & 99 & 139 & 13 & 79 & 19 & 97 & 23 & 103 & 370 & 20 & 107 & 12 & 285 & 28 & 72 & 13 & 7 & 1890 & $<20$ & 3 & 0.44 \\
\hline 137 & 54.4 & 12 & 105 & 141 & 14 & 80 & 22 & 99 & $<3$ & 107 & 384 & 22 & 113 & 10 & 301 & 29 & 58 & 12 & 8 & 2190 & $<20$ & 3 & 0.58 \\
\hline 142 & 56.4 & 13 & 84 & 145 & 10 & 71 & 16 & 60 & 20 & 93 & 377 & 19 & 148 & 12 & 303 & 19 & 67 & 6 & 11 & 3400 & $<20$ & $<3$ & 0.52 \\
\hline 147 & 58.4 & 11 & 96 & 130 & 11 & 72 & 18 & 72 & 13 & 98 & 374 & 16 & 82 & 10 & 275 & 44 & 60 & 10 & 14 & 2330 & $<20$ & $<3$ & 0.48 \\
\hline 152 & 60.4 & 11 & 95 & 137 & 10 & 74 & 28 & 90 & 18 & 99 & 375 & 19 & 118 & 9 & 312 & 41 & 60 & 7 & 2 & 2610 & $<20$ & 4 & 0.48 \\
\hline 157 & 62.4 & 10 & 85 & 154 & 11 & 73 & 27 & 81 & $<3$ & 96 & 425 & 23 & 160 & 8 & 304 & 22 & 70 & 8 & 14 & 5570 & $<20$ & 3 & 0.52 \\
\hline 162 & 64.4 & 13 & 86 & 141 & 10 & 73 & 12 & 61 & 16 & 90 & 361 & 18 & 132 & 10 & 273 & 9 & 73 & 2 & 3 & 4950 & $<20$ & 4 & 0.34 \\
\hline
\end{tabular}



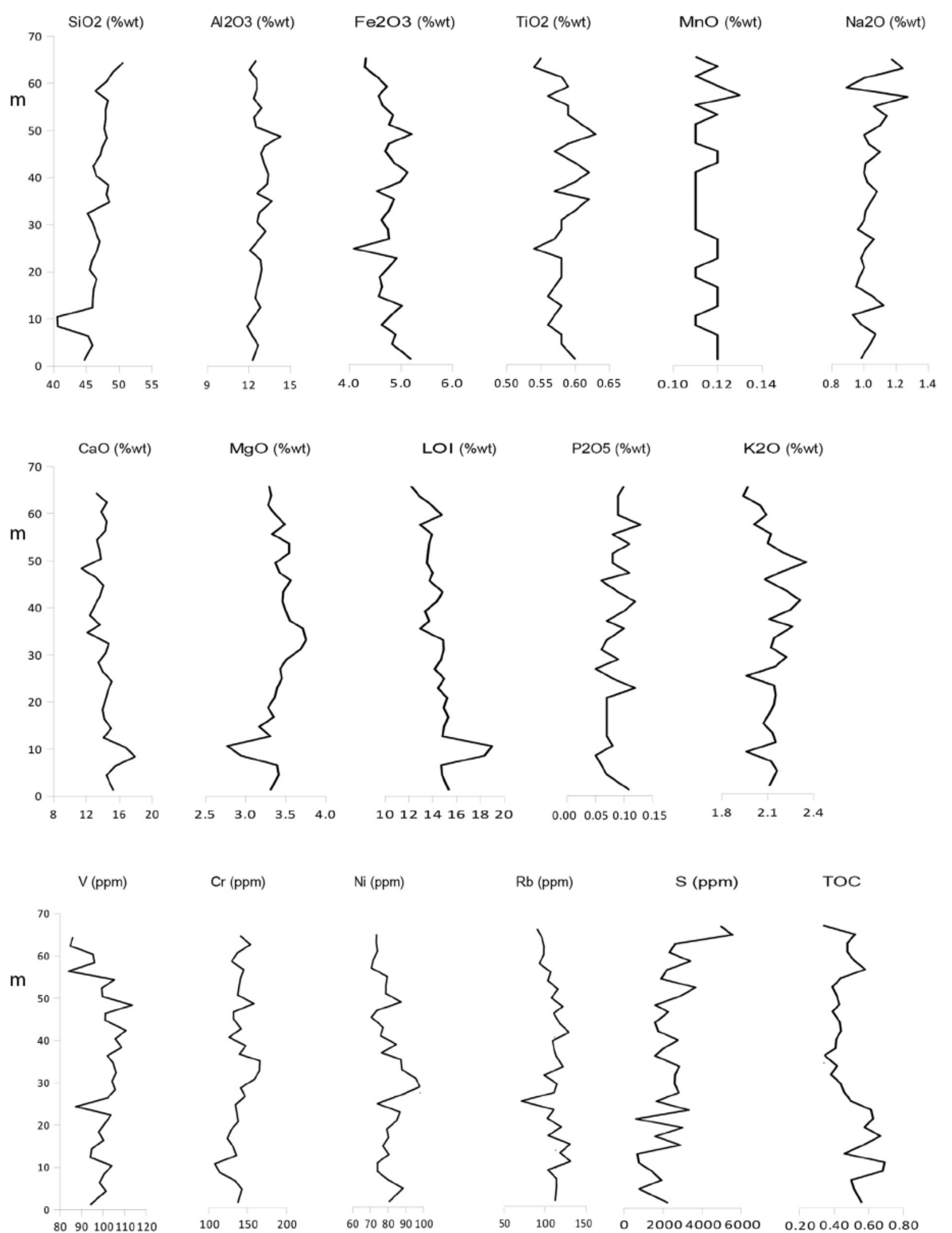

Figure 5. Along-section profiles of major oxides, LOI and minor elements in the sediment of the investigated succession, from the base ( $1.2 \mathrm{~m}$ ) to the top ( $64 \mathrm{~m}$ ).

of Geosciences and Georesources (CNR IGG) of Pisa and by J. Reitner (personal communication). The sampling for these analyses has been done slicing the carbonates and using a microdriller to collect the sub samples, in order to have precise measuring of the isotopes in the different areas. The powdered sub samples (100-200 $\mu \mathrm{g}$ ) were reacted for $10 \mathrm{~min}$ at $90^{\circ} \mathrm{C}$ with $100 \%$ phosphoric acid on an automated carbonate device connected to a VG PRISM mass spectrometer calibrated with NBS18, 

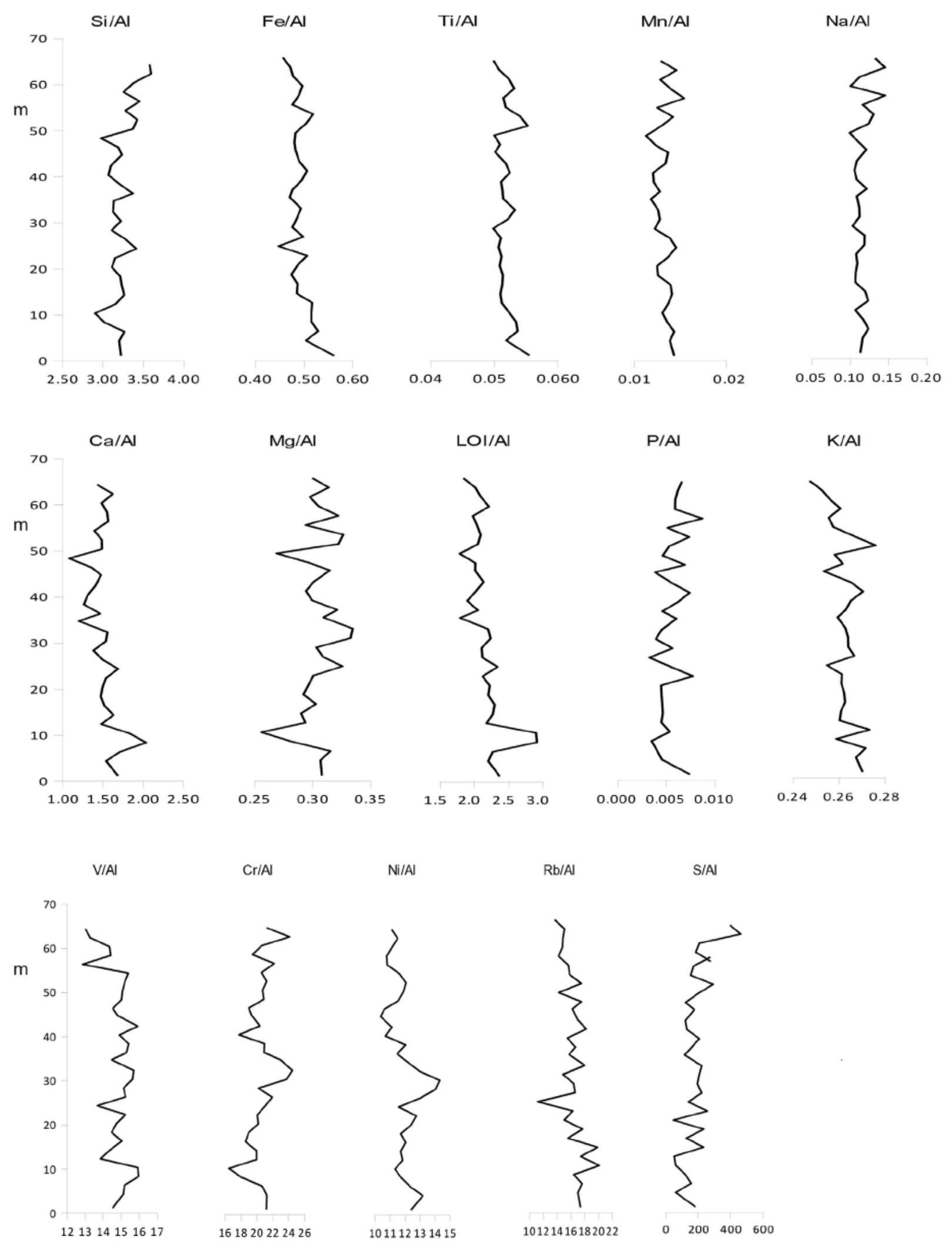

S/AI

Figure 6. Along-section profiles of elements occurring in the sediment normalized respect to Aluminium.

NBS19 and NBS20 standards. The results are reported in the conventional $\delta \%$ notation with reference to VPDB (Vienna Peedee Belemnite). For the dolomite samples (sample with N $20 \%$ of dolomite) $\delta^{18} \mathrm{O}$ values were calculated with the Rosenbaum and
Sheppard fractionation factor. Analytical reproducibility of the method, based on repeated analysis of standards is better than $\pm 0.1 \%$ for both carbon and oxygen (Rosenbaum, 1994; Rosenbaum and Sheppard, 1986). 
Table 4

Mineral abundances in the carbonates concretions.

\begin{tabular}{|c|c|c|c|}
\hline \multirow[t]{2}{*}{ Sample } & \multirow{2}{*}{$\frac{\text { Calcite }}{(\%)}$} & \multirow{2}{*}{$\frac{\text { Dolomite }}{(\%)}$} & \multirow{2}{*}{$\frac{\text { Detrital }}{(\%)}$} \\
\hline & & & \\
\hline En5 8A & 0 & 65 & 35 \\
\hline En5 8B & 6 & 57 & 37 \\
\hline En5 7A & 12 & 61 & 27 \\
\hline En5 7B & 0 & 66 & 34 \\
\hline En5 7C & 0 & 73 & 27 \\
\hline En5 6A & 5 & 58 & 37 \\
\hline En5 6B & 1 & 65 & 34 \\
\hline En5 6C & 5 & 60 & 35 \\
\hline En5 5A & 0 & 75 & 25 \\
\hline En5 5B & 0 & 74 & 26 \\
\hline En5 5C & 10 & 55 & 35 \\
\hline En5 4A & 0 & 78 & 22 \\
\hline En5 4B & 0 & 71 & 29 \\
\hline En5 4C & 13 & 46 & 41 \\
\hline En5 3A & 0 & 80 & 20 \\
\hline En5 3B & 0 & 80 & 20 \\
\hline En5 3C & 11 & 50 & 39 \\
\hline En5 2A & 0 & 82 & 18 \\
\hline En5 2B & 1 & 75 & 23 \\
\hline En5 2C & 6 & 60 & 34 \\
\hline En5 1A & 0 & 71 & 29 \\
\hline En10 1A & 0 & 76 & 24 \\
\hline En10 1B & 2 & 75 & 22 \\
\hline En10 2A & 0 & 81 & 19 \\
\hline $\mathrm{Cr} 2 \mathrm{~A}$ & 0 & 80 & 20 \\
\hline $\mathrm{Cr} 2 \mathrm{~B}$ & 4 & 71 & 25 \\
\hline $\mathrm{Cr} 3 \mathrm{~A}$ & 0 & 65 & 35 \\
\hline $\mathrm{Cr} 3 \mathrm{~B}$ & 11 & 52 & 37 \\
\hline
\end{tabular}

\section{Results}

\subsection{Hosting sediments geochemistry and mineralogy}

XRD analyses (Table 1) show ca. 21\% content of carbonate minerals in the sediments, mainly formed of calcite, dolomite is also present (1\%) but just in few samples located between $28.4 \mathrm{~m}$ and $64.4 \mathrm{~m}$ along the section, coupled with the appearance of protodolomite.

The bulk of these carbonates is made of biogenic residuals, mainly foraminifera and calcareous nannoplancton. The other minerals in the Argille Azzurre Fm. sediments of the Enza section are quartz (ca. 20\%), clay minerals as smectite and illite (ca. 18\%), feldspars (ca. 12\%), plagioclase (ca. 9\%); muscovite (ca. 8\%) and chlorite (ca. 7\%). The along section chemical profiles show a clear perturbation around $10 \mathrm{~m}$ (Tables 2 and 3; Fig. 5) where a positive peak of $\mathrm{CaO}$, LOI and in part TOC is matched by a negative peak of to $\mathrm{SiO}_{2}, \mathrm{Al}_{2} \mathrm{O}_{3}, \mathrm{Fe}_{2} \mathrm{O}_{3}$, and $\mathrm{K}_{2} \mathrm{O}$. This is the most evident occurrence, but as other minor peaks in the logs of these elements, reflect the biogenic carbonate/silicate proportion.

$\mathrm{MgO}$ displays a peculiar trend with a maxima in the central portion of the log (between 30 and $35 \mathrm{~m}$ ) and an increasing trend up section. $\mathrm{Cr}$ and $\mathrm{Ni}$ show a similar trend. TOC has a variable distribution with concentrations around $0.6 \%$ up to $22 \mathrm{~m}$, then a decrease around $0.4 \%$ up to $52 \mathrm{~m}$ and then an increase at the top of the section. The normalisation with respect to $\mathrm{Al}$ (Fig. 6) shows that the peaks of $\mathrm{SiO}_{2}, \mathrm{Al}_{2} \mathrm{O}_{3}, \mathrm{Fe}_{2} \mathrm{O}_{3}, \mathrm{~K} 2 \mathrm{O}, \mathrm{Cr}$ and $\mathrm{Ni}$ persist at 10, 25 and $50 \mathrm{~m}$.

Figure 6 shows peaks in the normalized profiles of $\mathrm{Cr}, \mathrm{Ni}$ and $\mathrm{Mg}$ between 30 and $40 \mathrm{~m}$.

\subsection{Carbonate geochemistry and mineralogy}

Carbonate bodies are mainly composed of dolomite (in average $68 \%$ bulk weight) with low and variable amounts of calcite (up to 13\%) (Table 4).

The detrital fraction (up to $41 \%$ ) is similar in all the samples: major components are quartz, feldspars, plagioclase, clay minerals and micas. The concretion $\mathrm{Cr} 3 \mathrm{~A}$ has also high content of pyrite and arsenopyrite (5\%) and is the only sample where strontianite is present (1\%).

Table 5

Major oxides in the carbonate concretions.

\begin{tabular}{|c|c|c|c|c|c|c|c|c|c|c|c|c|c|}
\hline \multirow[t]{2}{*}{ Sample } & $\mathrm{SiO}_{2}$ & $\mathrm{TiO}_{2}$ & $\mathrm{Al}_{2} \mathrm{O}_{3}$ & $\mathrm{Fe}_{2} \mathrm{O}_{3}$ & $\mathrm{MnO}$ & $\mathrm{MgO}$ & $\mathrm{CaO}$ & $\mathrm{Na}_{2} \mathrm{O}$ & $\mathrm{K}_{2} \mathrm{O}$ & $\mathrm{P}_{2} \mathrm{O}_{5}$ & LOI & $\mathrm{Mg} / \mathrm{Ca}$ & $\mathrm{Sr} / \mathrm{Ca}$ \\
\hline & (wt \%) & (wt \%) & (wt \%) & (wt \%) & (wt \%) & (wt \%) & (wt \%) & (wt \%) & (wt \%) & (wt \%) & (wt \%) & & \\
\hline En5 8A & 12.94 & 0.17 & 3.46 & 1.83 & 0.09 & 17.98 & 25.26 & 0.55 & 0.58 & 0.09 & 37.06 & 0.6007 & 0.0018 \\
\hline En5 8B & 21.53 & 0.26 & 6.04 & 2.68 & 0.13 & 13.32 & 23.47 & 0.66 & 0.94 & 0.09 & 30.87 & 0.4789 & 0.0018 \\
\hline En5 7A & 14.95 & 0.18 & 3.72 & 1.86 & 0.09 & 17.34 & 24.01 & 0.60 & 0.63 & 0.09 & 36.53 & 0.6094 & 0.0021 \\
\hline En5 7B & 14.47 & 0.18 & 4.02 & 1.78 & 0.10 & 17.52 & 24.56 & 0.60 & 0.62 & 0.08 & 36.08 & 0.6020 & 0.0016 \\
\hline En5 7C & 16.60 & 0.23 & 4.75 & 2.18 & 0.11 & 15.36 & 25.54 & 0.57 & 0.77 & 0.11 & 34.77 & 0.5075 & 0.0018 \\
\hline En5 6A & 13.34 & 0.19 & 3.67 & 1.84 & 0.09 & 18.02 & 24.91 & 0.52 & 0.63 & 0.06 & 36.73 & 0.6104 & 0.0017 \\
\hline En5 6B & 12.74 & 0.18 & 3.75 & 1.73 & 0.09 & 17.97 & 25.07 & 0.53 & 0.60 & 0.07 & 37.27 & 0.6049 & 0.0018 \\
\hline En5 6C & 16.94 & 0.20 & 4.22 & 2.16 & 0.11 & 15.63 & 24.59 & 0.60 & 0.72 & 0.10 & 34.73 & 0.5364 & 0.0023 \\
\hline En5 5A & 14.91 & 0.19 & 3.89 & 1.87 & 0.10 & 17.47 & 24.40 & 0.58 & 0.66 & 0.10 & 35.83 & 0.6042 & 0.0020 \\
\hline En5 5B & 16.54 & 0.21 & 4.24 & 2.00 & 0.10 & 16.82 & 24.05 & 0.59 & 0.74 & 0.12 & 34.58 & 0.5902 & 0.0022 \\
\hline En5 5C & 23.32 & 0.27 & 6.05 & 2.95 & 0.13 & 12.92 & 22.96 & 0.66 & 0.98 & 0.08 & 29.68 & 0.4748 & 0.0023 \\
\hline En5 4A & 14.52 & 0.18 & 3.72 & 1.81 & 0.10 & 17.65 & 24.67 & 0.54 & 0.63 & 0.11 & 36.07 & 0.6037 & 0.0018 \\
\hline En5 4B & 15.40 & 0.18 & 3.81 & 1.77 & 0.10 & 17.37 & 24.41 & 0.58 & 0.66 & 0.10 & 35.61 & 0.6005 & 0.0023 \\
\hline En5 4C & 24.95 & 0.29 & 6.41 & 3.23 & 0.15 & 11.84 & 23.08 & 0.72 & 1.03 & 0.14 & 28.15 & 0.4329 & 0.0019 \\
\hline En5 3A & 15.00 & 0.19 & 4.08 & 1.84 & 0.10 & 17.41 & 24.47 & 0.61 & 0.63 & 0.10 & 35.58 & 0.6004 & 0.0022 \\
\hline En5 3B & 17.01 & 0.19 & 3.92 & 1.89 & 0.10 & 16.80 & 24.32 & 0.66 & 0.68 & 0.11 & 34.33 & 0.5829 & 0.0019 \\
\hline En5 3C & 25.96 & 0.29 & 6.62 & 3.12 & 0.14 & 11.50 & 22.68 & 0.74 & 1.07 & 0.10 & 27.78 & 0.4279 & 0.0017 \\
\hline En5 2A & 13.08 & 0.18 & 3.77 & 1.69 & 0.09 & 17.80 & 25.11 & 0.54 & 0.62 & 0.08 & 37.03 & 0.5982 & 0.0020 \\
\hline En5 2B & 15.62 & 0.19 & 3.93 & 1.70 & 0.09 & 17.20 & 24.46 & 0.66 & 0.65 & 0.11 & 35.40 & 0.5934 & 0.0022 \\
\hline En5 2C & 22.81 & 0.26 & 5.92 & 2.80 & 0.14 & 12.48 & 24.01 & 0.74 & 0.94 & 0.09 & 29.84 & 0.4386 & 0.0020 \\
\hline En5 1A & 19.66 & 0.26 & 5.62 & 2.56 & 0.12 & 14.00 & 23.46 & 0.62 & 0.90 & 0.10 & 32.70 & 0.5036 & 0.0014 \\
\hline En10 1A & 14.79 & 0.20 & 4.36 & 1.78 & 0.10 & 17.13 & 23.90 & 0.37 & 0.57 & 0.21 & 36.59 & 0.5680 & 15.2939 \\
\hline En10 1B & 21.75 & 0.27 & 6.08 & 2.40 & 0.11 & 13.45 & 22.31 & 0.50 & 0.85 & 0.20 & 32.07 & 0.6585 & 11.1116 \\
\hline En10 2A & 16.78 & 0.21 & 4.59 & 2.05 & 0.11 & 16.07 & 23.85 & 0.45 & 0.61 & 0.23 & 35.05 & 0.4833 & 21.6196 \\
\hline $\mathrm{Cr} 2 \mathrm{~A}$ & 25.40 & 0.28 & 6.04 & 2.46 & 0.15 & 12.95 & 22.80 & 0.68 & 0.84 & 0.14 & 28.26 & 0.6379 & 6.0197 \\
\hline $\mathrm{Cr} 2 \mathrm{~B}$ & 26.93 & 0.24 & 5.80 & 2.12 & 0.11 & 14.04 & 21.32 & 0.82 & 0.73 & 0.23 & 27.66 & 0.7167 & 15.6611 \\
\hline $\mathrm{Cr} 3 \mathrm{~A}$ & 14.14 & 0.27 & 5.60 & 8.94 & 0.26 & 13.19 & 27.29 & 0.92 & 0.78 & 1.30 & 27.33 & 0.6029 & 18.8705 \\
\hline $\mathrm{Cr} 3 \mathrm{~B}$ & 25.47 & 0.27 & 5.27 & 2.60 & 0.14 & 16.86 & 26.43 & 0.73 & 0.75 & 0.32 & 21.16 & 0.6738 & 19.5430 \\
\hline
\end{tabular}


Dolomite content increases toward the internal parts of the chimneys, whereas in the external portion the calcite cement and the detrital fraction are more abundant. Terrigenous components, such as $\mathrm{SiO}_{2}, \mathrm{Al}_{2} \mathrm{O}_{3}$ and $\mathrm{Fe}_{2} \mathrm{O}_{3}$, increase towards the external areas; whereas, elements pertaining to the carbonate phases have their maximum in the middle and internal parts (Table 5; Fig. 7).

The trace elements $\mathrm{Cr}, \mathrm{V}, \mathrm{Ni}$ and $\mathrm{Rb}$ show maximum concentrations in the external parts of the chimney, while $S$ has a small
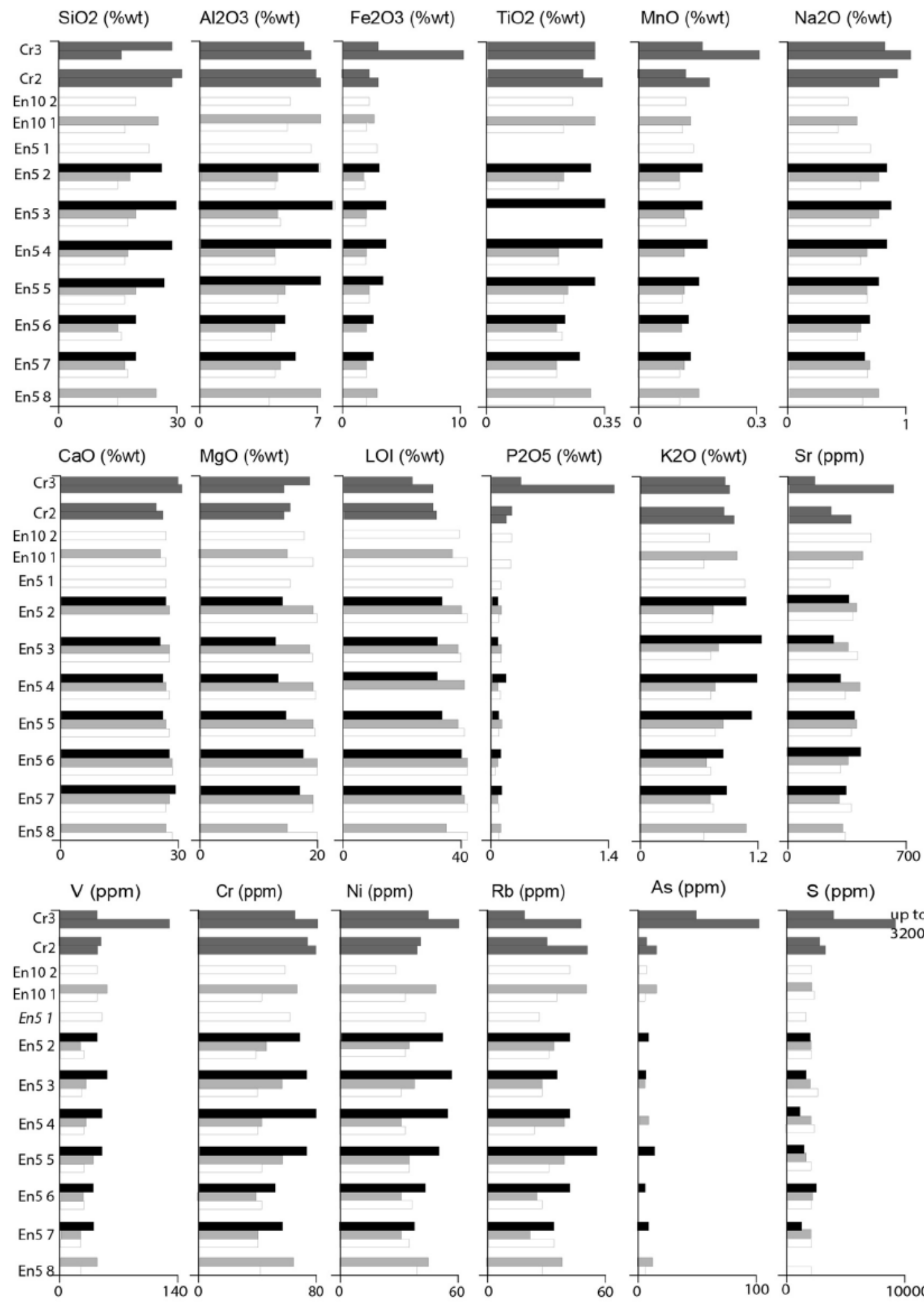

Chimney external part (C)

Chimney central part (B)

Chimney internal part (A)

Slabs: upper (B); lower (A)

Figure 7. Major oxides concentration, LOI and minor elements of the carbonate chimneys (En5 En10), slab ( $\mathrm{Cr} 2)$ and the concretion ( $\mathrm{Cr} 3)$. 
Table 6

Minor elements in the carbonate concretions.

\begin{tabular}{|c|c|c|c|c|c|c|c|c|c|c|c|c|c|c|c|c|c|c|c|}
\hline Sample & $\frac{\mathrm{V}}{(\mathrm{ppm})}$ & $\frac{\mathrm{Cr}}{(\mathrm{ppm})}$ & $\frac{\text { Co }}{(\mathrm{ppm})}$ & $\frac{\mathrm{Ni}}{(\mathrm{ppm})}$ & $\frac{\mathrm{Cu}}{(\mathrm{ppm})}$ & $\frac{\mathrm{Zn}}{(\mathrm{ppm})}$ & $\frac{\text { As }}{(\mathrm{ppm})}$ & $\frac{\mathrm{Rb}}{(\mathrm{ppm})}$ & $\frac{\mathrm{Sr}}{(\mathrm{ppm})}$ & $\frac{\mathrm{Y}}{(\mathrm{ppm})}$ & $\frac{\mathrm{Zr}}{(\mathrm{ppm})}$ & $\frac{\mathrm{Nb}}{(\mathrm{ppm})}$ & $\frac{\mathrm{Ba}}{(\mathrm{ppm})}$ & $\frac{\text { La }}{(\mathrm{ppm})}$ & $\frac{\mathrm{Ce}}{(\mathrm{ppm})}$ & $\frac{\mathrm{Pb}}{(\mathrm{ppm})}$ & $\frac{\text { Th }}{(\mathrm{ppm})}$ & $\frac{\mathrm{s}}{(\mathrm{ppm})}$ & $\frac{\text { TOC }}{(\%)}$ \\
\hline En5 8A & 24 & 35 & $<3$ & 34 & 6 & 34 & 5 & 23 & 320 & 2 & 2 & 2 & 223 & 12 & 23 & $<3$ & $<3$ & 1950 & 0.48 \\
\hline En5 8B & 40 & 56 & $<3$ & 39 & 4 & 26 & 10 & 32 & 308 & 3 & 17 & 7 & 300 & 12 & 40 & $<3$ & $<3$ & 1060 & 0.60 \\
\hline En5 7A & 22 & 36 & $<3$ & 30 & 4 & 32 & $<3$ & 30 & 356 & 3 & 2 & 6 & 264 & $<3$ & 22 & $<3$ & 6 & 2330 & 0.24 \\
\hline En5 7B & 21 & 36 & $<3$ & 27 & 12 & 29 & $<3$ & 18 & 286 & 3 & 2 & 5 & 235 & 11 & 47 & $<3$ & 7 & 2150 & 0.22 \\
\hline En5 7C & 36 & 51 & $<3$ & 33 & 12 & 40 & 10 & 29 & 332 & 2 & 2 & 6 & 269 & 4 & 39 & $<3$ & 5 & 1380 & 1.00 \\
\hline En5 6A & 26 & 39 & $<3$ & 32 & 7 & 34 & $<3$ & 25 & 301 & 2 & 2 & 8 & 250 & $<3$ & 25 & $<3$ & $<3$ & 2250 & 0.45 \\
\hline En5 6B & 24 & 35 & $<3$ & 27 & 14 & 33 & $<3$ & 22 & 331 & 2 & 2 & 5 & 258 & 11 & 31 & $<3$ & $<3$ & 2450 & 0.93 \\
\hline En5 6C & 33 & 43 & $<3$ & 38 & 10 & 39 & 5 & 36 & 413 & 2 & 2 & 6 & 250 & 10 & 53 & $<3$ & 7 & 2660 & 0.36 \\
\hline En5 5A & 27 & 37 & $<3$ & 31 & 9 & 38 & $<3$ & 26 & 349 & 5 & 2 & 5 & 245 & 9 & 44 & $<3$ & 3 & 2160 & 0.67 \\
\hline En5 5B & 32 & 50 & $<3$ & 30 & 14 & 37 & $<3$ & 33 & 384 & 4 & 2 & 6 & 273 & 9 & 37 & $<3$ & 4 & 1850 & 0.26 \\
\hline En5 5C & 44 & 65 & 4 & 45 & 10 & 51 & 13 & 48 & 380 & 7 & 19 & 9 & 278 & 18 & 37 & $<3$ & 7 & 1590 & 0.40 \\
\hline En5 4A & 26 & 35 & $<3$ & 29 & 10 & 35 & $<3$ & 21 & 310 & 4 & 2 & 6 & 243 & $<3$ & 28 & $<3$ & 4 & 2590 & 1.13 \\
\hline En5 4B & 27 & 38 & $<3$ & 27 & 6 & 35 & 7 & 34 & 397 & 3 & 2 & 6 & 259 & 4 & 25 & $<3$ & 5 & 2061 & 0.77 \\
\hline En5 4C & 45 & 70 & $<3$ & 47 & 17 & 50 & $<3$ & 36 & 310 & 3 & 6 & 5 & 296 & 13 & 33 & $<3$ & 7 & 1340 & 0.29 \\
\hline En5 3A & 23 & 35 & $<3$ & 27 & 8 & 31 & $<3$ & 24 & 376 & 5 & 2 & 4 & 272 & 9 & 25 & $<3$ & $<3$ & 2880 & 0.20 \\
\hline En5 3B & 27 & 48 & $<3$ & 33 & 6 & 36 & 4 & 24 & 334 & 5 & 2 & 3 & 258 & $<3$ & 33 & $<3$ & 5 & 2280 & 0.22 \\
\hline En5 3C & 50 & 64 & 4 & 49 & 19 & 51 & 7 & 30 & 269 & 6 & 16 & 5 & 259 & 6 & 54 & $<3$ & 8 & 1830 & 0.35 \\
\hline En5 2A & 26 & 34 & $<3$ & 29 & 10 & 35 & $<3$ & 26 & 365 & 2 & 2 & 6 & 223 & $<3$ & 31 & $<3$ & $<3$ & 2390 & 1.02 \\
\hline En5 2B & 20 & 40 & $<3$ & 30 & 6 & 33 & $<3$ & 29 & 388 & 2 & 4 & 4 & 251 & 3 & 32 & $<3$ & 10 & 1940 & 0.22 \\
\hline En5 2C & 40 & 61 & 4 & 44 & 21 & 47 & 8 & 36 & 352 & 10 & 13 & 6 & 284 & 26 & 43 & $<3$ & 4 & 1910 & 1.39 \\
\hline En5 1A & 42 & 55 & $<3$ & 37 & 17 & 44 & $<3$ & 23 & 239 & 5 & 2 & 4 & 255 & 12 & 37 & $<3$ & $<3$ & 1750 & 0.49 \\
\hline En10 1A & 39 & 36 & $<3$ & 30 & 14 & 16 & 4 & 30 & 374 & 6 & 17 & 5 & 202 & 10 & 32 & 12 & 4 & 2520 & \\
\hline En10 1B & 49 & 58 & $<3$ & 42 & 13 & 25 & 13 & 44 & 421 & 10 & 39 & 7 & 253 & 3 & 26 & 15 & $<3$ & 2270 & \\
\hline En10 2A & 37 & 52 & $<3$ & 25 & 9 & 16 & 5 & 36 & 466 & 10 & 34 & 5 & 251 & 9 & 13 & 11 & $<3$ & 2380 & \\
\hline Cr2A & 41 & 71 & $<3$ & 34 & 8 & 23 & 14 & 45 & 349 & 11 & 73 & 4 & 284 & $<3$ & 3 & 13 & $<3$ & 3560 & \\
\hline $\mathrm{Cr} 2 \mathrm{~B}$ & 46 & 67 & $<3$ & 35 & 8 & 22 & 5 & 27 & 237 & 7 & 65 & 3 & 201 & 3 & 26 & 10 & $<3$ & 3070 & \\
\hline Cr3A & 116 & 71 & 22 & 53 & 15 & 42 & 87 & 42 & 590 & 21 & 20 & 7 & 241 & 4 & 35 & 92 & $<3$ & 32,000 & \\
\hline $\mathrm{Cr} 3 \mathrm{~B}$ & 40 & 58 & 4 & 39 & 12 & 20 & 41 & 17 & 159 & 3 & 36 & $<3$ & 202 & $<3$ & $<3$ & 20 & $<3$ & 4390 & \\
\hline
\end{tabular}


increase in the internal areas. The overall content of trace elements is higher in samples En5 with respect to samples En10.

The slab (sample $\mathrm{Cr} 2$ ) and the concretion ( $\mathrm{Cr} 3)$ consist of dolomite (up to $80 \% \mathrm{wt}$ ), whereas calcite is present in correspondence of the white zone of $\mathrm{Cr} 2 \mathrm{~B}$ ( $4 \%$ wt) and in the carbonate fraction of $\mathrm{Cr} 3 \mathrm{~B}$ (11\% wt) (Table 4). MgO is usually higher in the chimneys than in the slabs. These latter, on the other hand, show higher concentrations of $\mathrm{V}, \mathrm{Cr}$, and $\mathrm{S}$.

$\mathrm{Cr} 3 \mathrm{~A}$ shows higher concentrations of trace elements than $\mathrm{Cr} 2$ and $\mathrm{Cr} 3 \mathrm{~B}$ (Fig. 7). For instance $\mathrm{S}$ reaches the concentration of $32,000 \mathrm{ppm}$ in $\mathrm{Cr} 3 \mathrm{~A}$ (Table 6). Furthermore, this sample has the lowest concentration of $\mathrm{SiO}_{2}$.

\subsection{Carbonate stable isotopes}

In Table 7 are presented the results for the $\delta^{13} \mathrm{C}$ and $\delta^{18} \mathrm{O}$ for carbonate samples. The slabs show carbon isotope values more depleted than chimneys, in particular $\mathrm{Cr} 3 \mathrm{~A}$ is the most negative with 40.13\% VPDB.

$\delta^{13} \mathrm{C}$ values are generally more negatives toward the internal parts (A) of the En5 chimney, ranging between 17.07 and $20.06 \%$ VPDB, respect to the $\delta^{13} \mathrm{C}$ of $8.6 \%$ VPDB, detected in the externals parts in the sample En5_3C. A further variation of ${ }^{13} \mathrm{C}$ has been observed from the top (En5_8) to the bottom (En5_1) of the chimney showing less negative $\delta^{13} \mathrm{C}$ values. The $\delta^{18} \mathrm{O}$ isotope values range between $4.37 \%$ and $6.24 \%$ VPDB. In this case the slabs values, ranging between $4.4 \%$ and $6.24 \%$ VPDB, are close to those from the chimneys, which are between $4.37 \%$ and $6.21 \%$ VPDB.

\subsection{Carbonate petrography}

Petrographic observation of thin sections was performed along En5 chimney, on the $\mathrm{Cr} 3 \mathrm{~A}$ dark concretion and on the carbonate breccia enclosed in the rhodolith rich layer.

- The EN5 chimney is cemented by dolomitic micrite (hereafter referred to as dolomicrite) and microsparite (Figs. 8A and 9A) with abundant angular to sub angular quartz clasts (up to $35 \%$

vol., 50-150 $\mu \mathrm{m}$ ). SEM EDS analyses revealed the presence of clusters of framboidal pyrite engulfed within the dolomitic cements (Fig. 9B). The chimney shows clear zonation probably due to dissolution-cementation cycles (Fig. $8 \mathrm{~B}-\mathrm{C}$ ). This zonation is marked by corrosion surfaces often lined by a thin generation (ca. $10 \mu \mathrm{m}$ ) of isopachous scalenohedral calcite (Fig. 8B-C). Locally, the chimneys are made of alternating peloidal micrite and dolomicrite. Peloids are 250-500 $\mu \mathrm{m}$ ovoidal to circular in shape, organized in clotted fabric (Fig. 8D); inter peloids porosity is filled with microcrystalline cements and sand sized clasts of quartz. Thin levels (ca. $500 \mu \mathrm{m}$ ) of sandy dolomicrite are breached by sigmoidal fractures (Fig. $8 \mathrm{E}-\mathrm{F}$ ). Well rounded clasts of glauconite (Figs. 8C and 9C) are sparse in the matrix as well as poorly rounded mica and plagioclase crystals. The chimneys are often breached by systems of radial and longitudinal fractures partially filled by microsparite (Fig. 8F). Some of those networks show geometrical intersections that suggest a likely affinity with tension cracks structures (Fig. 8G).

- Dark limestone (sample $\mathrm{Cr} 3 \mathrm{~A}$ ), which stratigraphically overlies the chimneys, is made of micrite with clasts of quartz ( $>50 \% \mathrm{vol}$,

$50-150 \mu \mathrm{m})$ and mottled micrite that shows peloidal fabric. Toward the upper surface of the slab, this facies is bounded by red coralline algae (Fig. $8 \mathrm{H}$ ). Rhodalgal coated chips of fossil barren mudstone together with foraminifera and coral re mains are shown in Figure 8I.

- In situ carbonate breccia is made of millimetre sized pebbles of micrite, micrite with abundant sand sized clasts of quartz and red algae fragments. Several centimetre sized rhodolithes were described within this facies (Fig. 8J). This facies show high voids ratio; sometimes voids are filled by mesosparite and botryoids of acicular aragonite (Fig. 8J). An isopachous generation of scalenohedral calcite growth on the carbonatic pebbles. Macro borings are restricted to the rhodolithes, indicating lithification before the settlement of macroborers (Fig. $8 \mathrm{~K}-\mathrm{L}$ ). Macroborings are filled with bright luminescence micrite, with sand sized clasts of quartz and probably feldspar (Fig. $8 \mathrm{~K}-\mathrm{L}$ )

\section{Discussion}

The chemical composition of the Argille Azzurre Fm. that hosts the seep carbonates reflects the mineralogy of the eroded rocks supplying sediments to the continental shelf during the Pleistocene (Capozzi and Picotti, 2010; Capozzi et al., 2012; Dinelli et al., 2012). Elements linked with quartz, plagioclase, micas, clay minerals and feldspars are abundant. The enrichment of $\mathrm{Ni}$ and $\mathrm{Cr}$ indicates the presence of mafic and ultramafic minerals, derived from the contribution of local magmatic rocks occurring within the drainage area in the emerging Northern Apennines chain (Picotti et al., 2007; Ghielmi et al., 2010; Gunderson et al., 2014). The enrichment in $\mathrm{CaO}$, $\mathrm{LOI}$ and $\mathrm{TOC}$ matched by a negative peak of $\mathrm{SiO}_{2}, \mathrm{Al}_{2} \mathrm{O}_{3}, \mathrm{Fe}_{2} \mathrm{O}_{3}$, and $\mathrm{K}_{2} \mathrm{O}$ at $10 \mathrm{~m}$ seems to be associated to the presence of a thin horizon probably corresponding to a peak of primary productivity, linked to calcareous phytoplankton and associated with organic carbon. Negative peaks of $\mathrm{Al}_{2} \mathrm{O}_{3}, \mathrm{Fe}_{2} \mathrm{O}_{3}, \mathrm{~K}_{2} \mathrm{O}, \mathrm{TiO}_{2}, \mathrm{~V}$ and $\mathrm{Rb}$ (Fig. 5), coupled with the positive peak of $\mathrm{Si} / \mathrm{Al}$ (Fig. 6) at $25 \mathrm{~m}$ suggest a grain size increase, corresponding to the occurrence of thin sandy and silty sand layers in the succession, coupled with the first occurrence of carbonate concretions. The same variations can be found at $50 \mathrm{~m}$, where the sandy layers are well developed and thicker slabs occur along the bedding planes (Oppo et al., in this isue). The carbonate minerals in the sediments are mainly attributed to the presence of fossil tests. However, dolomite and protodolomite occur only in the upper $35 \mathrm{~m}$ of the section, suggesting an origin linked to the same processes that generated the associated carbonate concretions.

The concretions are mainly composed of authigenic dolomite ( $>60 \% \mathrm{wt})$, variable amounts of calcite $(<15 \% \mathrm{wt})$ and detrital fraction. The cements petrography frequently shows clotted peloidal micrite. This microfabric has been widely observed in methane derived authigenic carbonates (MDAC) found in active or fossil cold seep systems (e.g. Peckmann et al., 2001; Magalhaes,

Table

Stable isotopes in the carbonate concretions. The symbol ${ }^{*}$ indicates the measurements performed at CNR-IGG in Pisa.

\begin{tabular}{|c|c|c|}
\hline \multirow[t]{2}{*}{ Sample } & $\delta^{13} \mathrm{C}$ & $\delta^{18} \mathrm{O}$ \\
\hline & (vpdb) & (vpdb) \\
\hline En5 8A & -20.06 & 5.86 \\
\hline En5 8B & -19.49 & 5.47 \\
\hline En5 6A & -19.31 & 6.2 \\
\hline En5 6B & -18.97 & 6.13 \\
\hline En5 4A* & -18.22 & 6.08 \\
\hline En5 3A & -18.61 & 6.21 \\
\hline En5 3C & -8.60 & 4.37 \\
\hline En5 1A & -17.07 & 5.59 \\
\hline En5 1B & -16.07 & 5.49 \\
\hline Gr2A* & -28.56 & 6.12 \\
\hline Gr3A & -35.42 & 4,49 \\
\hline $\mathrm{Cr} 3 \mathrm{~A}$ & -40.13 & 4.44 \\
\hline $\mathrm{Cr} 3 \mathrm{~A}^{*}$ & -30.69 & 5.10 \\
\hline Cr3B* (calcite) & -30.91 & 5.44 \\
\hline $\mathrm{Cr} 3 \mathrm{~B}^{*}$ & -35.39 & 6.13 \\
\hline $\mathrm{Cr} 3 \mathrm{~B}^{*}$ (calcite + dolomite) & -35.07 & 6.24 \\
\hline
\end{tabular}


2007; Capozzi et al., 2012). The peloids are interpreted as the product of intense and widespread microbial activity during carbonate precipitation (Peckmann et al., 2001; Peckmann and Thiel, 2004; Peckmann et al., 2007; Feng et al., 2008). Sulphur is particularly abundant in $\mathrm{Cr} 3 \mathrm{~A}$ and reflects the occurrence of pyrite and arsenopyrite. Further evidence of AOM can be found in the stable isotope ratios of the carbonate cements (Table 7). In the chimneys of Enza River, the $\delta^{13} \mathrm{C}$ ranges between $8.60 \%$ and $20.06 \%$ (VPDB), whereas the slabs have lower values, ranging between $28.56 \%$ and $40.13 \%$ VPDB. These values are indicative of AOM processes, as described for MDAC elsewhere (e.g. Rodriguez et al., 2000; Diaz del Rio et al., 2003; Hensen et al., 2007; Capozzi et al., 2012; Magalhaes et al., 2012). The $\delta^{18} \mathrm{O}$ in our samples vary from 4.4\% VPDB and 6.2\%。 VPDB meaning that they are significantly enriched in ${ }^{18} \mathrm{O}$ with respect to carbonates precipitated in a nowadays seawater conditions where $\delta^{18} \mathrm{O}$ is usually between $0 \%$ and 1\% VPDB (Magalhaes, 2007). This enrichment could be explained by the fact that carbonate dolomite phase shows usually an increase of $2-4 \%$ in $\delta^{18} \mathrm{O}$ composition respect to the ambient water (Budd, 1997; Aloisi et al., 2000; Oppo et al., in this issue); moreover according to Zachos et al., 2001 the values of $\delta^{18} \mathrm{O}$ during the Early Pleistocene were around 3\% VPDB (Oppo et al., in this
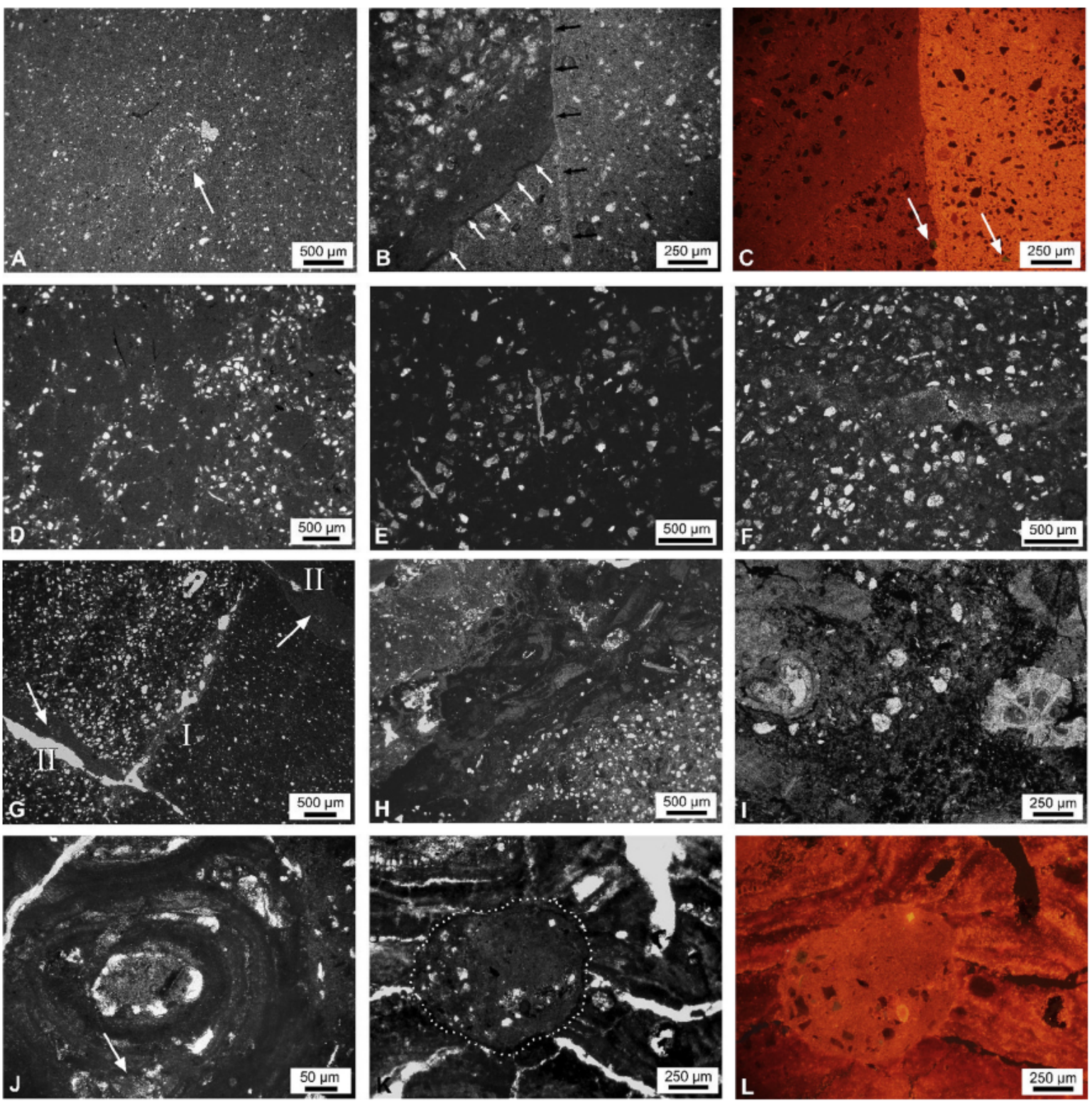

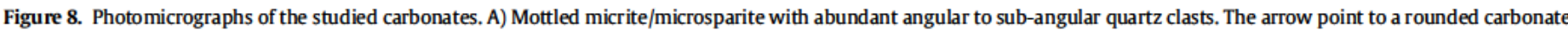

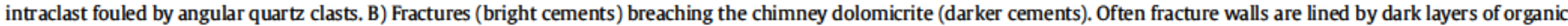

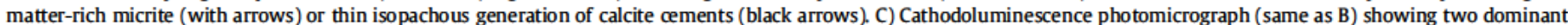

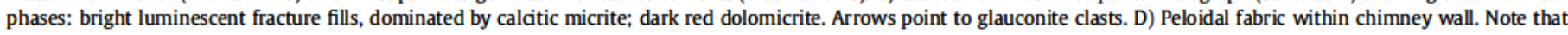

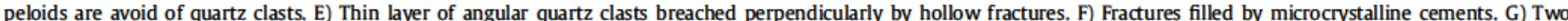

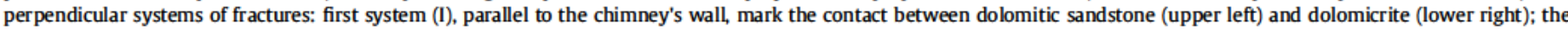

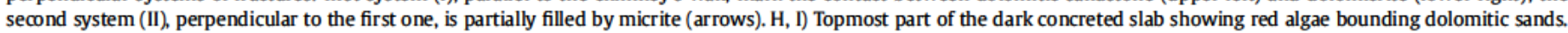

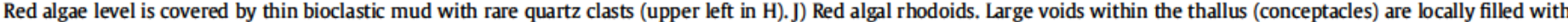

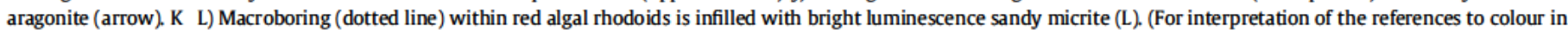
this figure legend, the reader is referred to the web version of this article.) 
issue). Petrographic analyses revealed that the chimneys are breached by expansion and sigmoidal fractures that have been described elsewhere within gas saturated sediments (Mazzini et al., 2005) and might be related to fluids expulsions related processes. This fracture network would represent the main mechanism of gas diffusion in fine grained sediments as observed in the model of Choi et al. (2011).

The progressive increase of carbonate cements is observed to ward the internal parts of the chimneys, which is mainly made of dolomite (up to $82 \%$ bulk rock in the sample En5_2A). Otherwise, authigenic calcite increases in the external parts. The higher content of calcite toward the edges reflects the increased influence of pore water rich of $\mathrm{SO}_{4}{ }^{2-}$, which inhibits the dolomite precipitation in anaerobic environment and favours the formation of calcite (e.g. Douglas, 2005).
Higher concentration of $\mathrm{SiO}_{2}$ and $\mathrm{Al}_{2} \mathrm{O}_{3}$ in the external areas of the EN5 evidence the gradual increase of siliciclastics from the internal to external areas, as suggested by the changes in mineralogy.

The chimneys form due to the focused fluids flow through a narrow discontinuity in the sediment interval consistent with the increase of sand within sedimentary record. The continued and vigorous flow progressively displaces the sediment particles and creates an open central vent that in some cases has been fully closed. The mainly dolomitic phase in cements suggests that all carbonate concretions so far analysed formed within the sediment pile near the Sulphate-Methane Interface (SMI) where the interstitial water is quite depleted in the sulphate component (Magalhaes, 2007; Magalhaes et al., 2012 and references therein).

The enrichment of $\mathrm{Fe}, \mathrm{S}, \mathrm{Ni}$ and $\mathrm{Cr}$, involved in the formation of sulphide minerals in the $\mathrm{Cr} 3 \mathrm{~A}$, could indicate precipitation/
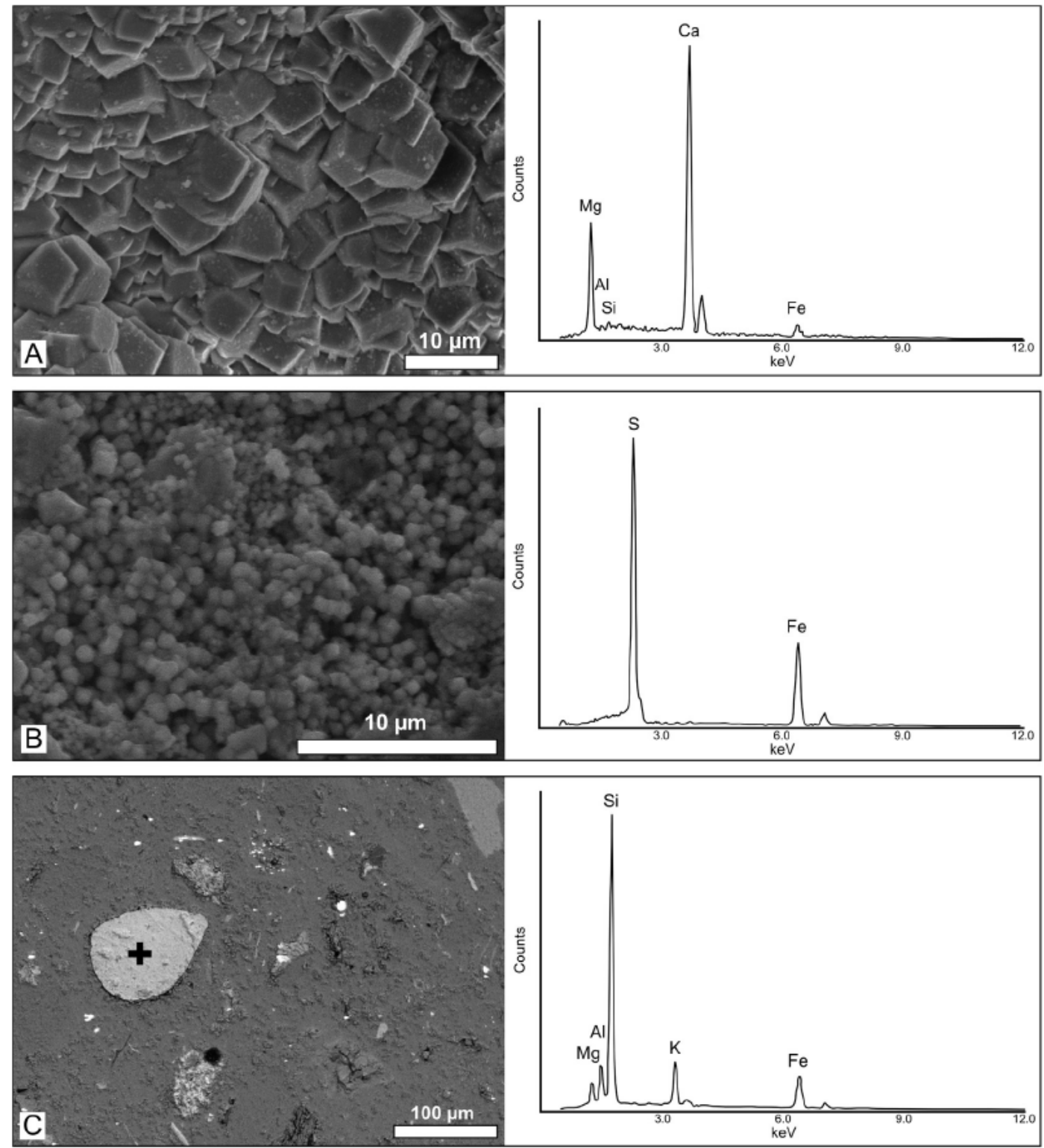

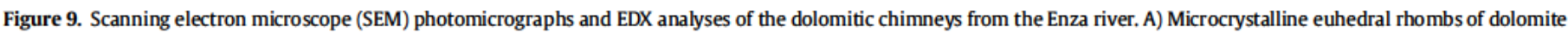

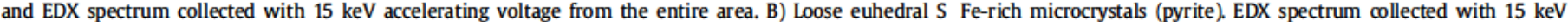

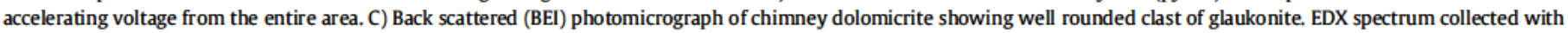
$15 \mathrm{keV}$ accelerating voltage from the bright rounded clast (cross). 
alteration of the carbonate by a renewed fluids leakage, permeating the rhodolith enriched layer, after a period of non deposition or erosion at the seafloor. The mottled micrite with its peloidal fabric indicates a renewed bacterial activity that possibly developed near the seafloor. In fact, in situ carbonate breccia also shows high voids ratio in same cases filled by mesosparite and botryoids of acicular aragonite that are very similar to those reported in methanederived authigenic carbonates recovered at the seafloor in the Black Sea and Adriatic Sea (Naudts et al., 2008; Capozzi et al., 2012).

\section{Conclusions}

Geochemical analyses on the Argille Azzurre Fm. sediments that host the carbonate concretions revealed their dominant siliciclastic composition, with small contents of biogenic calcite. Dispersed dolomite and protodolomite occur in the upper half of the section in association with the carbonate concretions.

Dolomite cement represents the $60-80 \%$ weight of the studied carbonate concretions. Therefore, the chimney palisade share compositional similarities with analogue dolomitic pipes identified in the Gulf of Cadiz (Magalhaes et al., 2012), southern New Zealand continental slope (Orpin, 1997), offshore Brazil (Wirsig et al., 2012), Black Sea (Reitner et al., 2005), Mediterranean Sea (Angeletti et al., in this issue) and other localities on land (e.g. De Boever et al., 2006a; Cau et al., in this issue).

Petrographic observations support the role of methane enriched fluids in the genesis of the carbonate chimneys and slabs. The occurrence of clotted and peloidal microfabric, which commonly represents bacterially induced precipitates, is frequently observed in methane derived authigenic carbonates. Moreover, stable carbon isotope ratios in cements indicate anaerobic oxida tion of methane and sulphate reduction during the carbonate precipitation.

The dolomite increase in the internal zone of chimneys reflects the progressive isolation from the $\mathrm{SO}_{4}$ rich pore water, which instead favours the calcite precipitation in the external area. The siliciclastic content increases moving from the internal to external zones due to the sediment displacement operated by the fluid flow along the central emission vent.

Pyrite, arsenopyrite and strontianite together with aragonite botryoids, possibly indicate secondary carbonate dissolution/precipitation within the rhodolith rich bed. The presence of this sul phide within carbonates points to a change in fluid leakage conditions within more permeable sediments saturated by $\mathrm{SO}_{4}$ rich marine water. These carbonates probably deposited after a period of seabed erosion in the Early Pleistocene shelf environment. The fluid leakage persists in present day, after the emersion of the Apennine foothills.

\section{Acknowledgements}

Funding was provided by the national project PRIN 2009 "Carbonate conduits linked to hydrocarbons enriched seepages" (PI R. Capozzi) of the Italian Ministry of University and Research (MIUR). Thanks are due to C. Mazzoli (University of Padua) for the access at the cathodoluminescence facilities. The authors are indebted with two anonymous reviewers for their constructive criticism. This is ISMAR Bologna scientific contribution number 1851.

\section{References}

Aloisi, G., Pierre, C., Rouchy, J.-M., Foucher, J.-P., Woodside, J., 2000. Methane-related

authigenic carbonates of eastern Mediterranean Sea mud volcanoes and their possible relation to gas hydrate destabilisation. Earth Planet. Sci. Lett. 184, 321338.
Angeletti, L, Canese, S., Franchi, F., Montagna, P., Reitner, J., Walliser, E.O., Taviani, M., 2015. The chimney forest of the deep Montenegrin margin, southeastern Adriatic Sea. In: Capozzi, R., Negri, A., Reithner, J., Taviani, M. (Eds.), Carbonate Conduits Linked to Hydrocarbon-enriched Fluid Escape. Special Issue of Mar. and Petr. Geo.

Boccaletti, M., Corti, G., Martelli, L, 2010. Recent and active tectonics of the external zone of the Northern Apennines (Italy). Earth Sci. 100,1331 1348.

Boetius, A., Ravenschlag, K., Schubert, C.J., Rickert, D., Widdel, F., Giesecke, A., Amann, R., Jorgensen, B.B., Witte, U., Pfannkuche, O., 2000. A marine microbial consortium apparently mediating anaerobic oxidation of methane. Nature 407 623626.

Budd, D.A., 1997. Cenozoic dolomites of carbonate islands: their attributes and origin. Earth Sci. Rev. 42, 147.

Capozzi, R., Picotti, V., 2010. Spontaneous fluid emissions in the Northern Apennines: geochemistry, structures and implications for the petroleum system. In: Goffey, G.P., Craig, J., Needham, T., Scott, R. (Eds.), Hydrocarbons in Contractional Belts, Geol. Soc London Spec. Publ. 348, London, pp. 115135.

Capozzi, R., Guido, F.L., Oppo, D., Gabbianelli, G., 2012. Methane-Derived Authigenic Carbonates (MDAC) in northern-central Adriatic Sea: relationships between reservoir and methane seepages. Mar. Geol. 332 334, 174188.

Capozzi, R., Negri, A., Reitner, J., Taviani, M., Franchi, F., Oppo, D. (Eds.), 2013.

Car-bonate Conduits Linked to Hydrocarbon-enriched Fluid Escape, Workshop and Field Seminar, Bologna, June 28th July 1st, 2013, Field Guide and Abstracts with Program, Bologna, pp. 151.

Cau, S., Franchi, F., Roveri, M., Taviani, M., 2015. The Pliocene-age Stirone river hydrocarbon chemoherm complex (Northern Apennines, Italy). In: Capozzi, R., Negri, A., Reithner, J., Taviani, M. (Eds.), Carbonate Conduits Linked to Hydrocarbon-enriched Fluid Escape. Special Issue of Marine and Petroleum Geology.

Choi, J., Mouillesseaux, K., Wang, Z, Fiji, H.D., Kinderman, S.S., Otto, G.W., Geisler, R. Kwon, O., Chen, J.N., 2011. Aplexone targets the HMG-CoA reductase pathway and differentially regulates arteriovenous angiogenesis. Development 138, 11731181.

Clari, P., Cavagna, S., Martire, L., Hunziker, J., 2004. A miocene mud volcano and its plumbing system: a chaotic complex revised (Monferrato, NW Italy). J. Sediment. Res. 74, 662676.

De Boever, E., Swennen, R., Dimitrov, L, 2006a. Lower Eocene carbonate-cemented "chimney" structures (Varna, Bulgaria) - control of seepage rates on their formation and stable isotopic signature. J. Geochem. Explor. 89, 7882.

De Boever, E., Swennen, R., Dimitrov, L., 2006b. Lower Eocene carbonate cemented chimneys (Varna, NE Bulgaria): formation mechanisms and the (a)biological mediation of chimney growth? Sediment. Geol. 185, 159173.

Díaz-del-Rio, V., Somoza, L., Martinez-Frias, J., Hernandez-Molina, F.J., Lunar, R., Fernandez-Puga, M.C., Maestro, A., Terrinha, P., Llave, E., Garcia, A., Garcia, A.C., Vazquez, J.T., 2001. Carbonate chimneys in the Gulf of Cadiz: initial report of their petrography and geochemistry. In: Akhmanov, G., Suzyumov, A. (Eds.), Geological Processes on Deep-water European Margins. IOC-UNESCO Workshop Report 175, pp. 5354.

Díaz-del-Río, V., Somoza, L, Martínez-Frías, J., Mata, P., Delgado, A., HernándezMolina, F.J., Lunar, R., Martín-Rubí, J.A., Maestro, A., Fernández-Puga, M.C., León, R., Lave, E., Medialdea, T., Vázquez, J.T., Hernández-Molina, F.J., 2003. Vast fields of hydrocarbon derived carbonate chimneys related to the accretionary wedge olistostrome of the Gulf of Cadiz. Mar. Geol. 195, 177200.

Dinelli, E., Ghosh, A., Rossi, V., Vaiani, S.C., 2012. Multiproxy reconstruction of Late Pleistocene-Holocene environmental changes in coastal successions: micro-fossil and geochemical evidences from the Po Plain (Northern Italy) Stratig-raphy 9, 153167.

Douglas, S., 2005. Mineralogical footprints of microbial life. Am. J. Sci. 305, 503525.

Feng, D., Chen, D.F., Qi, L., Roberts, H., 2008. Petrographic and geochemical characterization of seep carbonate from Alaminos Canyon, Gulf of Mexico. Chin. Sci.

Bull. SP Science in China Press 53 (11), 17161724

Franzini, M., Leoni, L, Saitta, M., 1972. A simple method to evaluate the matrix effects in X-Ray fluorescence analysis. X-Ray Spectrom. 1, 151154.

Franzini, M., Leoni, L, Saitta, M., 1975. Revisione di una metodologia analitica per fluorescenza-X, basata sulla correzione completa degli effetti di matrice. Rend

Soc. Ital. Mineral. Petrol. 31, 365378.

Ghielmi, M., Minervini, M., Nini, C., Rogledi, S., Rossi, M., Vignolo, A., 2010. Sedimentary and tectonic evolution in the eastern Po-Plain and northern Adriatic Sea area from Messinian to Middle Pleistocene (Italy). Rend. Lincei 21, S131 S166.

Goldsmith, J.R., Graf, D.L., 1958. Relation between lattice constants and composition of the Ca Mg carbonates. Am. Mineral. 43, 84101.

Gunderson, K.L., Pazzaglia, F.J., Picotti, V., Anastasio, D.A., Kodama, K.P., Rittenour, T., Frankel, K.F., Ponza, A., Berti, C., Negri, A., Sabbatini, A., 2014. Unraveling tectonic and climatic controls on synorogenic stratigraphy (Northern Apennines, Italy). GSA Bull. 126, 532552.

Hensen, C., Nuzzo, M., Hornibrook, E., Pinheiro, L.M., Bock, B., Magalhaes, V.H., Bruckmann, W., 2007. Sources of mud volcano fluids in the Gulf of Cadiz indications for hydrothermal imprint. Geochim. Cosmochim. Acta 71 (5), 1232 1248.

Lein, A. Yu, 2004. Authigenic carbonate formation in the ocean. Lithol. Miner. Resour. 39 (1), 1 30. Translated from Litologiya i Poleznye Iskopaemye 1, 2004: 3-35. 
Leoni, L., Saitta, M., 1976. X-ray fluorescence analysis of 29 trace elements in rock and mineral standards. Rend. Soc. Ital. Mineral. Petrol. 32, 497510

Leoni, L, Menichini, M., Saitta, M., 1982. Determination of S, Cl, and F in silicate rocks by X-Ray fluorescence analyses. X-Ray Spectrom. 11, 156158.

Lumsden, D.N., 1979. Discrepancy between thin-section and X-ray estimates of dolomite in limestone. J. Sediment. Res. 49, 429435.

Magalhaes, V.H., 2006 2007. Carbonates autigénicos e estruturas de escape de fluidos no Golfo de Cádis. Unpubl. Thesis (Tese apresentada do grau de Doutor em Geocencias.) Departamento de Geocencias, Universidade de Aveiro. a.a.

Magalhaes, V.H., Pinheiro, L.M., Ivanov, M.K., Kozlova, E., Blinova, V., Kolganova, J., Vasconcelos, C., McKenzie, J.A., Bernasconi, S.M., Kopf, A.J., Díaz-del-Río, V., González, F.J., Somoza, L., 2012. Formation processes of methane-derived authigenic carbonates from the Gulf of Cadiz. Sediment. Geol. 243244 , 155168

Mazzini, A., Aloisi, G., Akhmanov, G.G., Parnell, J., Cronin, B.T., Murphy, P., 2005. Integrated petrographic and geochemical record of hydrocarbon seepage on the

Vøring Plateau. J. Geol. Soc. Lond. 162, 815827.

Naudts, L, Greinert, J., Artemov, Y., Beaubien, S.E., Borowski, C, Batist, M.D., 2008. Anomalous sea-floor backscatter patterns in methane venting areas, Dnepr paleo-delta, NW Black Sea. Mar. Geol. 251, 253267.

Nyman, S.L., Nelson, C.S., Campbell, K.A., 2010. Miocene tubular concretions in East Coast Basin, New Zealand: analogue for the subsurface plumbing of cold seeps.

Mar. Geol, 272, 319336.

Oppo, D., Capozzi, R., Picotti, V., 2013. A new model of the petroleum system in the Northern Apennines, Italy. Mar. Pet. Geol. 48, 5776.

Oppo, D, Capozzi, R. Picotti, V . Ponza, A, 2015. A genetic model of hydrocarbonderived carbonate chimneys in shelfal fine grained sediments: the Enza River field, Northern Apennines (Italy). In: Capozzi, R., Negri, A., Reithner, J., Taviani, M. (Eds.), Carbonate Conduits Linked to Hydrocarbon-enriched Fluid Escape. Special Issue of Marine and Petroleum Geology.

Orpin, A.R., 1997. Dolomite chimneys as possible evidence of coastal fluid expulsion, uppermost Otago continental slope, southern New Zealand. Mar. Geol. 138,

5167.

Peckmann, J., Reimer, A., Luth, U., Luth, C, Hansen, B.T., Heinicke, C., Hoefs, J., Reitner, J., 2001. Methane-derived carbonates and authigenic pyrite from the northwestern Black Sea. Mar. Geol. 177, 129150.

Peckmann, J., Thiel, V., 2004. Carbon cycling at ancient methane-seeps. Chem. Geol. 205, 443467.

Peckmann, J., Campbell, K.A., Walliser, O.H., Reitner, J., 2007. A Late Devonian hydrocarbon-seep deposit dominated by dimerreloid brachiopods, Morocco. Palaios 22, 114122

Picotti, V., Capozzi, R., Bertozzi, G., Mosca, F., Sitta, A., Tornaghi, M., 2007. The Miocene petroleum system of the Northern Apennines in the central Po Plain
(Italy). In: Lacombe, O., Lavé, J., Roure, F., Vergés, J. (Eds.), Thrust Belts and Foreland Basins, from Fold Kinematics to Hydrocarbon System. Springer Verlag. Berlin, pp. 117131.

Pinheiro, L.M., Ivanov, M.K., Sautkin, A., Akhmanov, G., Magalhaes, V.H. Volkonskaya, A., Cunha, M.R., 2003. Mud volcanism in the Gulf of Cadiz: results from the TTR-10 cruise. Mar. Geol. 195, 131151.

Ponza, A., Pazzaglia, F.J., Picotti, V., 2010. Thrust-fold activity at the mountain front of the Northern Apennines (Italy) from quantitative landscape analysis. Geo-morphology 123, 211223.

Reitner, J., Peckmann, J., Reimer, A., Schumann, G., Thiel, V., 2005. Methane-derived carbonate build-ups and associated microbial communities at cold seeps on the lower Crimean shelf (Black Sea). Facies 51, 6679.

Rodriguez, N.M. Paull, C.K, Borowski, W.S, 2000. Zonation of authigenic carbonates within gas hydrate-bearing sedimentary sections on the Blake Ridge: offshore southeastern North America. Proc. ODP Sci. Results 164, 301312.

Rosenbaum, J., Sheppard, S.M.F., 1986. An isotopic study of siderites, dolomites and ankerites at high-temperatures. Geochim. Cosmochim. Acta 50 (6), 11471150

Rosenbaum, J.M., 1994. Stable-isotope fractionation between carbon-dioxide and calcite at $900{ }^{\circ} \mathrm{C}$. Geochim. Cosmochim. Acta 58 (17), 37473753.

Somoza, L., Díaz-del-Rio, V., Léon, R., Ivanov, M.K., Fernández-Puga, M.C., Lobato, A. Maestro, A., Hernández-Molina, F.J., Gardner, J.M., Rodero, J., Pinheiro, L.M. Vásquez, J.T., Medialdea, T., Fernández-Salas, L.M., 2003. Seabed morphology and hydrocarbon seepage in the Gulf of Cadiz mud volcano area: imagery of multibeam data and ultra-high resolution data. Mar. Geol. 195, 153176

Taviani, M., 2001. Fluid venting and associated processes. In: Vai, G.B., Maltini, I.P. (Eds.), Anatomy of an Orogen: the Apennines and Adjacent Mediterranean Basins. Kluwer Academic Publisher, Great Britain, pp. 351366.

Taviani, M., 2014. Marine chemosynthesis in the Mediterranean Sea. In: Goffredo, S. Baader, H., Dubinsky, Z. (Eds.), The Mediterranean Sea: Its History and Present Challenges. Springer, Science + Business Media Dordrecht, pp. 6983.

Teichert, B.M.A., Bohrmann, G., Suess, E., 2005. Chemoherms on Hydrate Ridge: unique microbially mediated carbonate build-ups growing into the water column. Palaeogeogr. Palaeoclimatol. Palaeoecol. 227, 6785.

Unterseh, S.L., 2013, May 6. Early Recognition of Seabed and Sub-seabed Natural Hydrocarbon Seeps in Deep Offshore Angola. Offshore Technology Conference.

Vanneste, H., Kastner, M., James, R.H., Connelly, D.P., Fisher, R.E., Kelly-Gerreyn, B.A. Heeschen, K., Haeckel, M., Mills, R.A., 2012. Authigenic carbonates from the Darwin Mud Volcano, Gulf of Cadiz: a record of palaeo-seepage of hydrocarbon bearing fluids. Chem. Geol. 300 301, 2439.

Wirsig, C., Kowsmann, R.O., Miller, D.J., de Oliveira Godoy, J.M., Mangini, A., 2012. U/ Th dating and post-depositional alteration of a cold seep carbonate chimney from the Campos Basin offshore Brazil. Mar. Geol. 329 331, 2433.

Zachos, J., Pagani, M., Sloan, L., Thomas, E., Billups, K., 2001. Trends, rhythms, and aberrations in global climate $65 \mathrm{Ma}$ to present. Science 292,686 693. 